\title{
Palladium(II)-Catalyzed Oxidative Transformation of Allylic Alcohols and Vinyl Ethers into 2-Alkoxytetrahydrofurans: Catechol as an Activator of Catalyst
}

Kimi Minami, Yasufumi Kawamura, Koichi Koga, and Takahiro Hosokawa*

\author{
Department of Environmental Systems Engineering, Kochi University of Technology, \\ 185 Miyanokuchi, Tosayamada, Kochi, 782-8502, Japan
}

\section{Supporting Information}

General: NMR spectra were recorded on a Varian UNITY INOVA 400 ('H NMR, 399.91 $\mathrm{MHz} ;{ }^{13} \mathrm{C} \mathrm{NMR}, 100.56 \mathrm{MHz}$ ) instrument for solutions in $\mathrm{CDCl}_{3}$ with $\mathrm{Me}_{4} \mathrm{Si}$ as an internal standard. Infrared spectra were measured on a JASCO FTIR-610 spectrometer. Elemental analyses were performed on Elementary VarioEL III. GLC analyses were performed on a Shimadzu GC-17A instrument by using DB-1 glass capillary column $(0.53 \mathrm{~mm} \times 30 \mathrm{~m})$ under the conditions of injection temperature $\left(200{ }^{\circ} \mathrm{C}\right)$, column temperature $\left(60-250{ }^{\circ} \mathrm{C}\right)$, and nitrogen gas pressure $\left(0.5 \mathrm{~kg} / \mathrm{cm}^{2}\right)$ with flame-ionization detectors and nitrogen as carrier gas. GC yields were determined using tetraethylene glycol dimethyl ether or anisole as an internal standard. Mass spectrum was obtained on a Shimadzu GCMS-QP5050 gas chromatograph-mass spectrometer at $0.7 \mathrm{eV}$ by using DB-1 glass capillary column $(0.25 \mathrm{~mm} \times 30 \mathrm{~m})$ under the conditions of injection temperature $\left(200{ }^{\circ} \mathrm{C}\right)$, column temperature $\left(60-250{ }^{\circ} \mathrm{C}\right)$, and helium gas pressure $\left(0.5 \mathrm{~kg} / \mathrm{cm}^{2}\right)$. All melting points were measured on Yanaco micro melting point apparatus and were uncorrected. Analytical thin layer chromatography (TLC) was performed with Merck silica gel $60 \mathrm{~F}_{254}$ plates. Column chromatography was performed with Merck silica gel 60.

Materials: Allyl alcohol, (E)-3-phenyl-2-propen-1-ol (cinnamyl alcohol), tetraethylene glycol dimethyl ether, and copper(II) acetate were commercially available from Wako Pure Chemical Industries, Ltd. $n$-Butyl vinyl ether, ethyl vinyl ether, catechol, and palladium(II) acetate were commercially available from Nakarai Tesque, Inc. (E)-3-(4-Nitrophenyl)-2-propen-1-ol (4-nitrocinnamyl alcohol) was obtained from Aldrich. 2-Buten-1-ol (crotyl alcohol) and 2-vinyloxytetrahydropyran were commercially available from TOKYO KASEI KOGYO, Co. Ltd. 
Synthesis of ( $Z$ )-4-Benzylidene-2-ethoxytetrahydrofuran (3a).

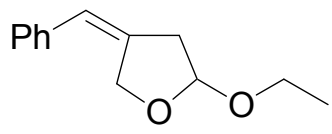

$3 a$

$\mathrm{Pd}(\mathrm{OAc})_{2}(11.2 \mathrm{mg}, 0.05 \mathrm{mmol}), \mathrm{Cu}(\mathrm{OAc})_{2}(9.1 \mathrm{mg}, 0.05 \mathrm{mmol})$, and catechol (11.0 mg, 0.1 mmol) were dissolved in $\mathrm{MeCN}(0.5 \mathrm{~mL})$ in a $25 \mathrm{~mL}$ side-armed round bottomed flask under $\mathrm{O}_{2}$ (balloon), and the mixture was stirred for $30 \mathrm{~min}$ at room temperature. Ethyl vinyl ether $(288 \mathrm{mg}, 4.0 \mathrm{mmol}$ ) was added to the flask, and a solution of (E)-3-phenyl-2-propen-1-ol (134 mg, $1.0 \mathrm{mmol})$ in $\mathrm{MeCN}(0.5 \mathrm{~mL})$ was then added. After the reaction mixture was stirred for $3 \mathrm{~h}$ at room temperature, GC yield of $3 \mathbf{a}$ was determined to be $84 \%$ by using tetraethylene glycol dimethyl ether as an internal standard. Isolation of pure 3a was made as follows. The same reaction as above was separately performed, and the reaction mixture was stirred for $3 \mathrm{~h}$. After filtration through Florisil column (10 mm x $80 \mathrm{~mm} 3 \mathrm{~g}$, EtOAc / $n$-hexane $=1 / 20)$, the solvent was evaporated under reduced pressure. The product $\mathbf{3 a}$ nearly pure was obtained in $82 \%$ yield (168 $\mathrm{mg}, 0.82 \mathrm{mmol}$ ), and further purification was made by thin-layer chromatography on silica gel.

3a :

Rf : $0.62($ EtOAc / $n$-hexane $=1 / 20)$.

bp (bulb-to-bulb) : $145-146{ }^{\circ} \mathrm{C}(1 \mathrm{mmHg})$.

1H NMR (400 MHz, $\left.\mathrm{CDCl}_{3}\right) \delta 1.21(\mathrm{t}, \mathrm{J}=7.1 \mathrm{~Hz}, 3 \mathrm{H}), 2.72(\mathrm{~d}, \mathrm{~J}=16.2 \mathrm{~Hz}, 1 \mathrm{H}), 2.94(\mathrm{dd}$, $\mathrm{J}=16.2,5.1 \mathrm{~Hz}, 1 \mathrm{H}), 3.50(\mathrm{dq}, \mathrm{J}=9.3,7.1 \mathrm{~Hz}, 1 \mathrm{H}), 3.77$ (dq, J= 9.3, $7.1 \mathrm{~Hz}, 1 \mathrm{H}), 4.68$ (d, $\mathrm{J}=2.1 \mathrm{~Hz}, 1 \mathrm{H}), 4.69(\mathrm{~d}, \mathrm{~J}=2.1 \mathrm{~Hz}, 1 \mathrm{H}), 5.23(\mathrm{~d}, \mathrm{~J}=5.1 \mathrm{~Hz}, 1 \mathrm{H}), 6.42(\mathrm{~s}, 1 \mathrm{H}), 7.12(\mathrm{~d}, \mathrm{~J}=$ $7.2 \mathrm{~Hz}, 2 \mathrm{H}), 7.19$ (t, J= $7.2 \mathrm{~Hz}, 1 \mathrm{H}), 7.33$ (t, J= 7.2 Hz, 2H).

${ }^{13} \mathrm{C}$ NMR (101 MHz, $\left.\mathrm{CDCl}_{3}\right) \delta$ 15.14, 41.12, 62.57, 67.85, 101.93, 121.55, 126.40, 127.78, 128.39, 137.34, 138.98.

FTIR (neat, $\mathrm{cm}^{-1}$ ) : 3053, 3025, 1739, 1598, 1184, 1095, 1032, 997.

GCMS m/e $204\left(\mathrm{M}^{+}\right)$.

Anal. Calcd for $\mathrm{C}_{13} \mathrm{H}_{16} \mathrm{O}_{2}$ : C, 76.42; H, 7.89. Found: C, 76.32; H, 7.49. 
Synthesis of (Z)-4-Benzylidene-2- $n$-buthoxytetrahydrofuran (3b).

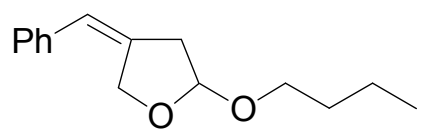

3b

$\mathrm{Pd}(\mathrm{OAc})_{2}(11.2 \mathrm{mg}, 0.05 \mathrm{mmol}), \mathrm{Cu}(\mathrm{OAc})_{2}(9.1 \mathrm{mg}, 0.05 \mathrm{mmol})$, and catechol (11.0 mg, $0.05 \mathrm{mmol}$ ) were dissolved in $\mathrm{MeCN}(0.5 \mathrm{~mL})$ in a $25 \mathrm{~mL}$ side-armed round bottomed flask under $\mathrm{O}_{2}$ (balloon), and the mixture was stirred for $30 \mathrm{~min}$ at room temperature. $n$-Buthyl vinyl ether $(401 \mathrm{mg}, 4.0 \mathrm{mmol})$ was added to the flask, and a solution of (E)-3-phenyl-2-propen-1-ol (134 mg, $1.0 \mathrm{mmol})$ in MeCN $(0.5 \mathrm{~mL})$ was then added. After the reaction mixture was stirred for $3 \mathrm{~h}$ at room temperature, the mixture was filtered through Florisil column (10 $\mathrm{mm} \times 80 \mathrm{~mm} 3 \mathrm{~g}, n$-hexane), and the solvent was evaporated under reduced pressure to give the product $3 \mathbf{b}(173 \mathrm{mg}, 0.74 \mathrm{mmol}, 74 \%)$ in nearly pure form. Further purification was made by thin-layer chromatography on silica gel.

$3 \mathbf{b}:$

Rf : $0.67($ EtOAc $/ n$-hexane $=1 / 9)$.

bp (bulb-to-bulb) : $173-175{ }^{\circ} \mathrm{C}(5 \mathrm{mmHg})$.

${ }^{1} \mathrm{H}$ NMR $\left(400 \mathrm{MHz}, \mathrm{CDCl}_{3}\right) \delta 0.91(\mathrm{t}, \mathrm{J}=7.5 \mathrm{~Hz}, 3 \mathrm{H}), 1.36$ (tq, J= 8.4, 7.5 Hz, 2H), 1.56 (tt, $\mathrm{J}=8.4,6.6 \mathrm{~Hz}, 2 \mathrm{H}), 2.71(\mathrm{~d}, \mathrm{~J}=16.4,1 \mathrm{H}), 2.93(\mathrm{dd}, \mathrm{J}=16.4,5.1 \mathrm{~Hz}, 1 \mathrm{H}), 3.44(\mathrm{dt}, \mathrm{J}=9.5$, $6.6 \mathrm{~Hz}, 1 \mathrm{H}), 3.71(\mathrm{dt}, \mathrm{J}=9.5,6.6 \mathrm{~Hz}, 1 \mathrm{H}), 4.68(\mathrm{~d}, \mathrm{~J}=2.3 \mathrm{~Hz}, 1 \mathrm{H}), 4.69(\mathrm{~d}, \mathrm{~J}=2.3 \mathrm{~Hz}, 1 \mathrm{H})$, $5.21(\mathrm{~d}, \mathrm{~J}=5.1 \mathrm{~Hz}, 1 \mathrm{H}), 6.42(\mathrm{~s}, 1 \mathrm{H}), 7.13(\mathrm{~d}, \mathrm{~J}=7.3 \mathrm{~Hz}, 2 \mathrm{H}), 7.20(\mathrm{t}, \mathrm{J}=7.3 \mathrm{~Hz}, 1 \mathrm{H}), 7.34$ (t, J= $7.3 \mathrm{~Hz}, 2 \mathrm{H}$ ).

${ }^{13} \mathrm{C}$ NMR (101 MHz, $\left.\mathrm{CDCl}_{3}\right) \delta$ 13.86, 19.34, 31.70, 41.09, 67.04, 67.87, 102.15, 121.51, 126.42, 127.81, 128.44, 137.40, 139.12.

FTIR (neat, $\mathrm{cm}^{-1}$ ) : 3026, 2930, 2870, 1599, 1492, 1449, 1423, 1345, 1180, 1097, 1038, 925, 840, 749, 695 .

GCMS m/e $232\left(\mathrm{M}^{+}\right)$.

Anal. Calcd for $\mathrm{C}_{15} \mathrm{H}_{20} \mathrm{O}_{2}: \mathrm{C}, 77.55 ; \mathrm{H}, 8.68$. Found: $\mathrm{C}, 77.53 ; \mathrm{H}, 8.62$. 
Synthesis of 2-Ethoxy-(Z)-4-(4-nitrobenzylidene)tetrahydrofuran (3c).

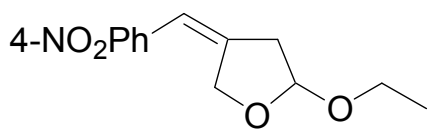

$3 c$

$\mathrm{Pd}(\mathrm{OAc})_{2}(11.2 \mathrm{mg}, 0.05 \mathrm{mmol}), \mathrm{Cu}(\mathrm{OAc})_{2}(9.1 \mathrm{mg}, 0.05 \mathrm{mmol})$, and catechol (11.0 mg, 0.1 mmol) were dissolved in $\mathrm{MeCN}(1.0 \mathrm{~mL})$ in a $25 \mathrm{~mL}$ side-armed round bottomed flask under $\mathrm{O}_{2}$ (balloon), and the mixture was stirred for $30 \mathrm{~min}$ at room temperature. Ethyl vinyl ether $(288 \mathrm{mg}, 4.0 \mathrm{mmol})$ was added to the flask, and (E)-3-(4-nitrophenyl)-2-propen-1-ol (179 mg, $1.0 \mathrm{mmol}$ ) was then added. After the reaction mixture was stirred for $12 \mathrm{~h}$ at room temperature, the mixture was filtered through Florisil column (10 mm x $130 \mathrm{~mm} 4 \mathrm{~g}$, EtOAc), and the solvent was evaporated under reduced pressure to gave 3c (241 $\mathrm{mg}, 0.96 \mathrm{mmol}, 96 \%$ yield) in solid state. Purification was made by recrystallization from hexane and EtOAc $(5: 1)$.

3c :

$\mathrm{mp}: 75-76{ }^{\circ} \mathrm{C}$.

${ }^{1} \mathrm{H}$ NMR (400 MHz, $\left.\mathrm{CDCl}_{3}\right) \delta 1.21(\mathrm{t}, \mathrm{J}=7.1 \mathrm{~Hz}, 3 \mathrm{H}), 2.80(\mathrm{~d}, \mathrm{~J}=16.9 \mathrm{~Hz}, 1 \mathrm{H}), 2.98(\mathrm{dd}$, $\mathrm{J}=16.9,4.9 \mathrm{~Hz}, 1 \mathrm{H}), 3.52(\mathrm{dq}, \mathrm{J}=8.5,7.1 \mathrm{~Hz}, 1 \mathrm{H}), 3.78(\mathrm{dq}, \mathrm{J}=8.5,7.1 \mathrm{~Hz}, 1 \mathrm{H}), 4.71(\mathrm{~d}$, $\mathrm{J}=2.4 \mathrm{~Hz}, 1 \mathrm{H}), 4.72(\mathrm{~d}, \mathrm{~J}=2.4 \mathrm{~Hz}, 1 \mathrm{H}), 5.27(\mathrm{~d}, \mathrm{~J}=4.9 \mathrm{~Hz}, 1 \mathrm{H}), 6.51(\mathrm{~s}, 1 \mathrm{H}), 7.20(\mathrm{~d}, \mathrm{~J}=$ $8.9 \mathrm{~Hz}, 2 \mathrm{H}), 8.20(\mathrm{~d}, \mathrm{~J}=8.9 \mathrm{~Hz}, 2 \mathrm{H})$.

${ }^{13} \mathrm{C} \mathrm{NMR}\left(101 \mathrm{MHz}, \mathrm{CDCl}_{3}\right.$ ) $\delta$ 15.13, 41.64, 62.81, 67.81, 101.87, 120.01, 123.88, 128.24, 143.67, 144.96, 145.93.

FTIR (nujol, cm-1) : 2927, 1652, 1592, 1513, 1099, 1049, 996.

GCMS m/e $249\left(\mathrm{M}^{+}\right)$.

Anal. Calcd for $\mathrm{C}_{13} \mathrm{H}_{15} \mathrm{NO}_{4}$ : C, 62.64; H, 6.07; N, 5.62. Found: C, 62.64; H, 6.07; N, 5.50 . 
Synthesis of (Z)-4-Benzyliden-2-tetrahydropyranyloxytetrahydrofuran (3d).

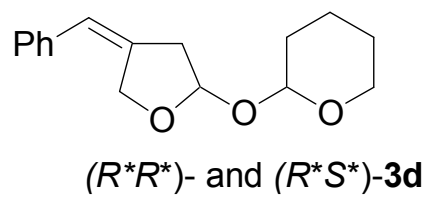

$\mathrm{Pd}(\mathrm{OAc})_{2}(22.5 \mathrm{mg}, 0.1 \mathrm{mmol}), \mathrm{Cu}(\mathrm{OAc})_{2}(18.2 \mathrm{mg}, 0.1 \mathrm{mmol})$, and catechol (22.0 mg, 0.2 $\mathrm{mmol}$ ) were dissolved in $\mathrm{MeCN}(0.5 \mathrm{~mL})$ in a $25 \mathrm{~mL}$ side-armed round bottomed flask under $\mathrm{O}_{2}$ (balloon), and the mixture was stirred for $30 \mathrm{~min}$ at room temperature. 2-Vinyloxytetrahydropyran (513 $\mathrm{mg}, 4.0 \mathrm{mmol}$ ) was added to the flask, and a solution of (E)-3-phenyl-2-propen-1-ol (134 mg, $1.0 \mathrm{mmol})$ in MeCN $(0.5 \mathrm{~mL})$ was then added. After the reaction mixture was stirred for $6 \mathrm{~h}$ at room temperature, the mixture was filtered through Florisil column (10 mm x $80 \mathrm{~mm} 3 \mathrm{~g}$, EtOAc $/ n$-hexane $=1 / 20)$, and the solvent was evaporated under reduced pressure to give a mixture of $R^{\star} R^{*}$ - and $R^{\star} S^{*}$-3d. Isolation of each isomer by thin-layer chromatography gave $R^{*} R^{*}$-3d (164 mg, $0.63 \mathrm{mmol})$ and $R^{\star} S^{*}$-3d (24 mg, $\left.0.09 \mathrm{mmol}\right)$. The product yield of $\mathbf{3 d}$ thus corresponds to $72 \%\left(R^{\star} R^{\star} / R^{\star} S^{\star}=87 / 13\right)$. The configuration of $R^{\star} R^{\star}$ and $R^{\star} S^{\star}$ was assigned on the bases of X-ray analysis of the corresponding $R^{\star} R^{\star}$-3e (see page $\mathrm{S} 11$ ).

$R * R * 3 \mathrm{~d}:$

Rf : $0.5($ EtOAc $/ n$-hexane $=1 / 4)$

bp (bulb-to-bulb) : $176-178{ }^{\circ} \mathrm{C}(4 \mathrm{mmHg})$.

${ }^{1} \mathrm{H}$ NMR $\left(400 \mathrm{MHz}, \mathrm{CDCl}_{3}\right) \delta 1.47-1.61(\mathrm{~m}, 4 \mathrm{H}), 1.66-1.74(\mathrm{~m}, 1 \mathrm{H}), 1.76-1.86(\mathrm{~m}, 1 \mathrm{H})$, $2.77(\mathrm{~d}, \mathrm{~J}=16.6,1 \mathrm{H}), 2.98$ (dd, J= 16.6, $4.96 \mathrm{~Hz}, 1 \mathrm{H}), 3.51-3.57(\mathrm{~m}, 1 \mathrm{H}), 3.85-3.92$ (m, 1H), $4.68(\mathrm{~d}, \mathrm{~J}=14.4 \mathrm{~Hz}, 1 \mathrm{H}), 4.70(\mathrm{~d}, \mathrm{~J}=14.4 \mathrm{~Hz}, 1 \mathrm{H}), 4.95(\mathrm{~s}, 1 \mathrm{H}), 5.27$ (d, J= $5.6 \mathrm{~Hz}$, $1 \mathrm{H}), 6.44(\mathrm{~s}, 1 \mathrm{H}), 7.13(\mathrm{~d}, \mathrm{~J}=7.8 \mathrm{~Hz}, 2 \mathrm{H}), 7.20(\mathrm{t}, \mathrm{J}=7.8 \mathrm{~Hz}, 1 \mathrm{H}), 7.33(\mathrm{t}, \mathrm{J}=7.8 \mathrm{~Hz}, 2 \mathrm{H})$. ${ }^{13} \mathrm{C}$ NMR (101 MHz, $\left.\mathrm{CDCl}_{3}\right) \delta$ 19.90, 25.36, 30.66, 40.76, 63.25, 68.07, 94.78, 97.86, 121.62, 126.45, 127.77, 128.44, 137.34, 138.88.

FTIR (neat, $\mathrm{cm}^{-1}$ ) : 3036, 2942, 2858, 1718, 1713, 1492, 1449, 1350, 1272, 1201, 1118, 1074 .

GCMS m/e $260\left(\mathrm{M}^{+}\right)$.

$R * S^{*} 3 \mathrm{~d}:$

Rf : $0.4($ EtOAc $/ n$-hexane $=1 / 4)$.

bp (bulb-to-bulb) : $132-135{ }^{\circ} \mathrm{C}(1 \mathrm{mmHg})$. 
${ }^{1} \mathrm{H}$ NMR $\left(400 \mathrm{MHz}, \mathrm{CDCl}_{3}\right) \delta$ 1.46-1.66 (m, 4H), 1.67-1.76 (m, 1H), 1.76-1.85 (m, 1H), $2.77(\mathrm{~d}, \mathrm{~J}=16.3,1 \mathrm{H}), 2.97(\mathrm{dd}, \mathrm{J}=16.3,4.56 \mathrm{~Hz}, 1 \mathrm{H}), 3.52-3.59(\mathrm{~m}, 1 \mathrm{H}), 3.94-4.01$ (m, $1 \mathrm{H}), 4.71(\mathrm{~d}, \mathrm{~J}=14.2 \mathrm{~Hz}, 1 \mathrm{H}), 4.83(\mathrm{~d}, \mathrm{~J}=14.2 \mathrm{~Hz}, 1 \mathrm{H}), 4.85(\mathrm{~s}, 1 \mathrm{H}$ ), 5.45 (d, J= $5.4 \mathrm{~Hz}$, $1 \mathrm{H}), 6.42(\mathrm{~s}, 1 \mathrm{H}), 7.13(\mathrm{~d}, \mathrm{~J}=7.7 \mathrm{~Hz}, 2 \mathrm{H}), 7.20(\mathrm{t}, \mathrm{J}=7.7 \mathrm{~Hz}, 1 \mathrm{H}), 7.33(\mathrm{t}, \mathrm{J}=7.7 \mathrm{~Hz}, 2 \mathrm{H})$.

\section{Synthesis of (Z)-4-(4-Nitrobenzyliden)-2-tetrahydropyranyloxytetrahydrofuran (3e).}

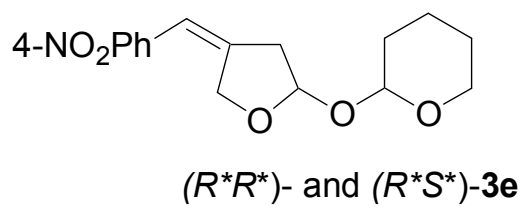

$\mathrm{Pd}(\mathrm{OAc})_{2}(11.2 \mathrm{mg}, 0.05 \mathrm{mmol}), \mathrm{Cu}(\mathrm{OAc})_{2}(9.1 \mathrm{mg}, 0.05 \mathrm{mmol})$, and catechol (11.0 mg, $0.1 \mathrm{mmol}$ ) were dissolved in $\mathrm{MeCN}(1.0 \mathrm{~mL})$ in a $25 \mathrm{~mL}$ side-armed round bottomed flask under $\mathrm{O}_{2}$ (balloon), and the mixture was stirred for $30 \mathrm{~min}$ at room temperature. 2-Vinyloxytetrahydropyran (513 $\mathrm{mg}, 4.0 \mathrm{mmol}$ ) was added to the flask, and (E)-3-(4-nitrophenyl)-2-propen-1-ol (179 mg, $1.0 \mathrm{mmol}$ ) was then added. After the reaction mixture was stirred for the $24 \mathrm{~h}$ at room temperature, the mixture was filtered through Florisil column $(10 \mathrm{~mm} \times 60 \mathrm{~mm} 2.5 \mathrm{~g}$, EtOAc $/ n$-hexane $=1 / 20)$, and the solvent was evaporated under reduced pressure to give a mixture of $R^{\star} R^{\star}$ - and $R^{\star} S^{*}$-3e. Isolation of each isomer by thin-layer chromatography gave $R^{*} R^{\star}-3 \mathrm{e}$ (205 mg, 0.67 $\mathrm{mmol})$ in solid state and $R^{\star} S^{*}-3 \mathrm{e}(35 \mathrm{mg}, 0.11 \mathrm{mmol})$ as liquid. The product yield of $3 \mathrm{e}$ thus corresponds to $78 \%\left(R^{\star} R^{\star} / R^{\star} S^{*}=86 / 14\right)$. Purification was made by recrystallization from hexane and diethyl ether $(3: 1)$. The configuration of $R^{*} R^{*}$ and $R^{\star} S^{\star}$ was assigned on the basis of X-ray analysis of $R^{\star} R^{\star}$-3e (see page $\mathrm{S} 11$ ).

$R^{\star} R^{\star}-3 \mathrm{e}$ :

Rf : $0.35($ EtOAc $/ n$-hexane $=1 / 4)$.

mp : $92-93{ }^{\circ} \mathrm{C}$.

${ }^{1} \mathrm{H} \mathrm{NMR}\left(400 \mathrm{MHz} \mathrm{CDCl}_{3}\right)$ ) $1.47-1.63(\mathrm{~m}, 4 \mathrm{H}), 1.68-1.77(\mathrm{~m}, 1 \mathrm{H}), 1.79-1.87(\mathrm{~m}, 1 \mathrm{H})$, $2.83(\mathrm{~d}, \mathrm{~J}=16.8,1 \mathrm{H}), 3.03(\mathrm{dd}, \mathrm{J}=16.8,4.9 \mathrm{~Hz}, 1 \mathrm{H}), 3.52-3.59(\mathrm{~m}, 1 \mathrm{H}), 3.86-3.92(\mathrm{~m}, 1 \mathrm{H})$, $4.59(\mathrm{~d}, 12.8 \mathrm{~Hz}, 1 \mathrm{H}), 4.63(\mathrm{~d}, 12.8 \mathrm{~Hz}, 1 \mathrm{H}), 4.95(\mathrm{~s}, 1 \mathrm{H}), 5.65(\mathrm{~d}, \mathrm{~J}=4.9 \mathrm{~Hz}, 1 \mathrm{H}), 6.53(\mathrm{~s}$, $1 \mathrm{H}), 7.26(\mathrm{~d}, \mathrm{~J}=8.76 \mathrm{~Hz}, 2 \mathrm{H}), 8.21(\mathrm{~d}, \mathrm{~J}=8.76 \mathrm{~Hz}, 2 \mathrm{H})$.

${ }^{13} \mathrm{C}$ NMR (101 $\left.\mathrm{MHz}, \mathrm{CDCl}_{3}\right) \delta$ 19.83, 25.31, 30.59, 41.23, 63.26, 68.00, 94.82, 97.74, 120.07, 123.87, 128.21, 143.62 144.83, 145.88.

FTIR (KBr, cm-1) : 3078, 2955, 2929, 1657, 1593, 1340, 1119, 989, 973, 924, 749, 688. 
GCMS m/e $305\left(\mathrm{M}^{+}\right)$.

Anal. Calcd for $\mathrm{C}_{16} \mathrm{H}_{19} \mathrm{NO}_{5}: \mathrm{C}, 62.94 ; \mathrm{H}, 6.27 ; \mathrm{N}, 4.59$. Found: C, 62.87; H, 6.57; N, 4.49 .

$R * S^{*} 3 \mathrm{e}:$

$\mathrm{Rf}: 0.24($ EtOAc $/ n$-hexane $=1 / 4)$.

1H NMR (400 MHz, $\left.\mathrm{CDCl}_{3}\right) \delta$ 1.48-1.64 (m, 4H), 1.67-1.78 (m, 1H), 1.78-1.86 (m, 1H), $2.83(\mathrm{~d}, \mathrm{~J}=16.6 \mathrm{~Hz} 1 \mathrm{H}$, ), $3.03(\mathrm{dd}, \mathrm{J}=16.6,4.5 \mathrm{~Hz}, 1 \mathrm{H}), 3.52-3.63(\mathrm{~m}, 1 \mathrm{H}), 3.95-4.06$ (m, $1 \mathrm{H}), 4.75(\mathrm{~d}, \mathrm{~J}=14.3 \mathrm{~Hz}, 1 \mathrm{H}), 4.88(\mathrm{~d}, \mathrm{~J}=14.3 \mathrm{~Hz}, 1 \mathrm{H}), 4.91(\mathrm{~s}, 1 \mathrm{H}), 5.50$ (d, J= $4.5 \mathrm{~Hz}$, 1H), $6.53(\mathrm{~s}, 1 \mathrm{H}), 7.45(\mathrm{~d}, \mathrm{~J}=8.9 \mathrm{~Hz}, 2 \mathrm{H}), 8.20(\mathrm{~d}, \mathrm{~J}=8.9 \mathrm{~Hz}, 2 \mathrm{H})$.

Synthesis of 2- $n$-Buthoxy-4-methylenetetrahydrofuran (3f).

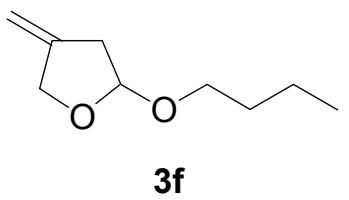

$\mathrm{Pd}(\mathrm{OAc})_{2}(11.2 \mathrm{mg}, 0.05 \mathrm{mmol}), \mathrm{Cu}(\mathrm{OAc})_{2}(9.1 \mathrm{mg}, 0.05 \mathrm{mmol})$, and catechol $(11.0 \mathrm{mg}$, $0.1 \mathrm{mmol}$ ) were dissolved in $\mathrm{MeCN}(0.5 \mathrm{~mL})$ in a $25 \mathrm{~mL}$ side-armed round bottomed flask under $\mathrm{O}_{2}$ (balloon), and the mixture was stirred for $30 \mathrm{~min}$ at room temperature. $n$-Butyl vinyl ether (401 mg, $4.0 \mathrm{mmol}$ ) was added to the flask, and a solution of allyl alcohol $(58 \mathrm{mg}, 1.0 \mathrm{mmol})$ in $\mathrm{MeCN}(0.5 \mathrm{~mL})$ was then added. After the reaction mixture was stirred for $3 \mathrm{~h}$ at room temperature, GC yield of $3 \mathrm{f}$ was determined to be $74 \%$ by using tetraethylene glycol dimethyl ether as an internal standard. The reaction mixture was filtered through Florisil column (17 mm x $80 \mathrm{~mm} 6 \mathrm{~g}, n$-hexane), and the solvent was evaporated under reduced pressure to give $3 \mathrm{f}$ ( $115 \mathrm{mg}, 0.73 \mathrm{mmol}$, $73 \%$ yield) in nearly pure form.

3f :

bp (bulb-to-bulb) : $54-55{ }^{\circ} \mathrm{C}(4 \mathrm{mmHg})$.

${ }^{1} \mathrm{H} \mathrm{NMR}\left(400 \mathrm{MHz}, \mathrm{CDCl}_{3}\right) \delta 0.83$ (t, J= 7.5 Hz, 3H), 1.29 (tq, J= 8.4, 7.5 Hz, 2H), 1.47 (tt, $\mathrm{J}=8.4,6.6 \mathrm{~Hz}, 2 \mathrm{H}), 2.43(\mathrm{~d}, \mathrm{~J}=16.3 \mathrm{~Hz}, 1 \mathrm{H}), 2.61(\mathrm{dd}, \mathrm{J}=16.3,5.2 \mathrm{~Hz}, 1 \mathrm{H}), 3.33(\mathrm{dt}, \mathrm{J}=$ 9.3, 7.5 Hz, 1H), $3.61(\mathrm{dt}, \mathrm{J}=9.3,7.5 \mathrm{~Hz}, 1 \mathrm{H}), 4.26(\mathrm{~d}, \mathrm{~J}=12.4 \mathrm{~Hz}, 1 \mathrm{H}), 4.31(\mathrm{~d}, \mathrm{~J}=12.4$ $\mathrm{Hz}, 1 \mathrm{H}), 4.98(\mathrm{~s}, 1 \mathrm{H}), 5.03(\mathrm{~s}, 1 \mathrm{H}), 5.11(\mathrm{~d}, \mathrm{~J}=5.2 \mathrm{~Hz}, 1 \mathrm{H})$.

${ }^{13} \mathrm{C}$ NMR (101 MHz, $\left.\mathrm{CDCl}_{3}\right) \delta$ 13.65, 19.18, 31.55, 39.16, 66.75, 68.90, 103.50, 104.55, 
145.64 .

FTIR (neat, $\mathrm{cm}^{-1}$ ) : 3081, 2960, 2871, 1714, 1671, 921, 883, 735.

GCMS m/e $156\left(\mathrm{M}^{+}\right)$.

Synthesis of 2-n-Butoxy-4-vinyltetrahydrofuran (3g).

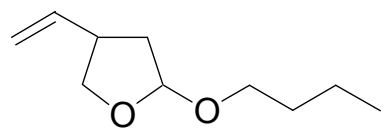

$3 g$

$\mathrm{Pd}(\mathrm{OAc})_{2}(11.2 \mathrm{mg}, 0.05 \mathrm{mmol}), \mathrm{Cu}(\mathrm{OAc})_{2}(9.1 \mathrm{mg}, 0.05 \mathrm{mmol})$, and catechol (11.0 mg, 0.1 $\mathrm{mmol})$ were dissolved in $\mathrm{MeCN}(0.5 \mathrm{~mL})$ in a $25 \mathrm{~mL}$ side-armed round bottomed flask under $\mathrm{O}_{2}$ (balloon), and the mixture was stirred for $30 \mathrm{~min}$ at room temperature. $n$-Buthyl vinyl ether $(401 \mathrm{mg}, 4.0 \mathrm{mmol})$ was added to the flask, and a solution of 2-buten-1-ol (trans/cis $=5 / 1,72 \mathrm{mg}, 1.0 \mathrm{mmol})$ in $\mathrm{MeCN}(0.5 \mathrm{~mL})$ was then added. After the reaction mixture was stirred for $3 \mathrm{~h}$ at room temperature, GC yield of $\mathbf{3 g}$ was determined to be $74 \%$ by using anisole as an internal standard. The reaction mixture was filtered through Florisil column (11 mm x $80 \mathrm{~mm} 2.8 \mathrm{~g}$, EtOAc, $100 \mathrm{~mL}$ ), and the solvent was evaporated under reduced pressure to give a 71:29 mixture of trans ${ }^{-}$and $c i s-3 g$. The ratio was determined by NMR. Purification was made by bulb-to-bulb distillation.

$3 g$ :

bp (bulb-to-bulb) : $102-105{ }^{\circ} \mathrm{C}(4 \mathrm{mmHg})$.

${ }^{1} \mathrm{H} \mathrm{NMR}\left(400 \mathrm{MHz}, \mathrm{CDCl}_{3}\right) \delta 0.87$ (t, J= $7.3 \mathrm{~Hz}, 0.9 \mathrm{H},[$ trans] $), 0.88(\mathrm{t}, \mathrm{J}=7.3 \mathrm{~Hz}, 2.1 \mathrm{H}$, $[$ cis] $), 1.33(\mathrm{sex}, \mathrm{J}=7.3 \mathrm{~Hz}, 0.6 \mathrm{H},[$ trans $]$ ), 1.33 (sex, J= 7.3 Hz, 1.4H, [cis]), 1.61 (ddd, J= 13.4, 8.8, $2.9 \mathrm{~Hz}, 0.7 \mathrm{H},[\mathrm{cis}]$ ), 1.70 (ddd, J=14.4, 8.5, $5.2 \mathrm{~Hz}, 0.3 \mathrm{H}$, [trans]), 2.02 (ddd, J= 14.4, 8.5 Hz, 0.3H, [trans]), 2.32 (ddd, J= 13.4, 8.8, $5.9 \mathrm{~Hz}, 0.7 \mathrm{H},[$ cis]), 2.75 (sex, J= 8.5 $\mathrm{Hz}, 0.7 \mathrm{H},[\mathrm{cis}]), 3.02(\mathrm{sex}, \mathrm{J}=8.3 \mathrm{~Hz}, 0.3 \mathrm{H},[$ trans $]), 3.28(\mathrm{dt}, \mathrm{J}=9.6,7.3 \mathrm{~Hz}, 0.3 \mathrm{H}$, [trans]), $3.30(\mathrm{dt}, \mathrm{J}=9.5,7.3 \mathrm{~Hz}, 0.7 \mathrm{H},[$ cis] $), 3.55(\mathrm{dd}, \mathrm{J}=8.8,8.8 \mathrm{~Hz}, 0.7 \mathrm{H},[$ cis $]), 3.55$ (dd, J= 8.5, 8.5 Hz, 0.3H, [trans]), 3.65 (dt, J= 9.6, 7.3 Hz, 0.3H, [trans]), 3.66 (dt, J= 9.5, $7.3 \mathrm{~Hz}, 0.7 \mathrm{H},[c i s]), 3.92(\mathrm{~m}, 1 \mathrm{H}), 3.92(\mathrm{dd}, \mathrm{J}=8.8,8.8 \mathrm{~Hz}, 0.7 \mathrm{H},[c i s]), 4.05$ (dd, J= 8.5, $8.5 \mathrm{~Hz}, 0.3 \mathrm{H},[$ trans] $), 4.93(\mathrm{dd}, \mathrm{J}=10.0,1.8 \mathrm{~Hz}, 0.7 \mathrm{H},[c i s]), 4.95$ (dd, J= 10.0, $1.6 \mathrm{~Hz}$, $0.3 \mathrm{H},[c i s]), 5.01$ (dd, J= 17.0, $1.8 \mathrm{~Hz}, 0.7 \mathrm{H},[$ cis $]$ ), 5.04 (dd, J= 17.0, $1.6 \mathrm{~Hz}, 0.3 \mathrm{H},[$ trans $]$ ), $5.07(\mathrm{~d}, \mathrm{~J}=5.2 \mathrm{~Hz}, 0.3 \mathrm{H},[$ trans $]$ ), 5.09 (dd, J= 5.9, $2.9 \mathrm{~Hz}, 0.7 \mathrm{H},[$ cis $]$ ), 5.65 (ddd, J= 17.0, 
$10.0,8.5 \mathrm{~Hz}, 0.3 \mathrm{H},[\operatorname{trans}]), 5.81$ (ddd, J= 17.0, 10.0, $8.8 \mathrm{~Hz}, 0.7 \mathrm{H},[$ cis] $)$.

${ }^{13} \mathrm{C} \mathrm{NMR}$ (101 MHz, $\mathrm{CDCl}_{3}$ ) $\delta$ 13.79, 19.28 [trans], 19.30 [cis], 31.73 [trans], 31.76 [cis], 39.21 [cis], 39.40 [trans], 41.42 [trans], 43.04 [cis], 66.95 [trans], 67.36 [cis], 70.82 [cis] , 71.69 [trans], 103.98 [trans], 104.45 [cis], 115.12 [trans], 115.30 [cis], 138.82 [cis], 139.09 [trans].

FTIR (neat, cm-1) : 3081, 2960, 2880, 1643, 1347, 1098, 917, 665.

GCMS m/e $170\left(\mathrm{M}^{+}\right)$.

\section{D-NOESY Experiment of cis and trans $3 \mathrm{~g}$.}

The $400 \mathrm{MHz}$ proton NMR spectra were acquired in $5 \mathrm{~mm}$ tubes on approximately 2 wt\% solutions in $\mathrm{CDCl}_{3}$ on a Varian Unity Plus instrument using a Varian PFD Indirect Probe. As shown below, the H-4 proton of cis isomer $3 \mathrm{~g}$ showed a correlation with cis proton of $\mathrm{H}-3$. The proton $\mathrm{H}-3$ also indicated a correlation with the adjacent $\mathrm{H}-2$ proton. In the case of trans $\mathbf{3 g}$, the $\mathrm{H}-4$ proton similarly showed a correlation with $c$ is proton of $\mathrm{H}^{-}-3$, but no correlation was observed between this $\mathrm{H}^{-}-3$ proton and the adjacent $\mathrm{H}-2$ proton. These facts allowed us to assign the cis/trans configuration of $\mathbf{3 g}$ successfully. The 2D-NOESY spectrum obtained is shown in Figure S-1 (see next page).

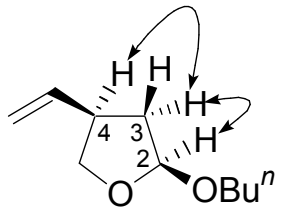

cis-3g

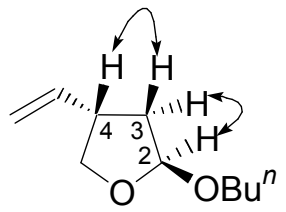

trans-3g 


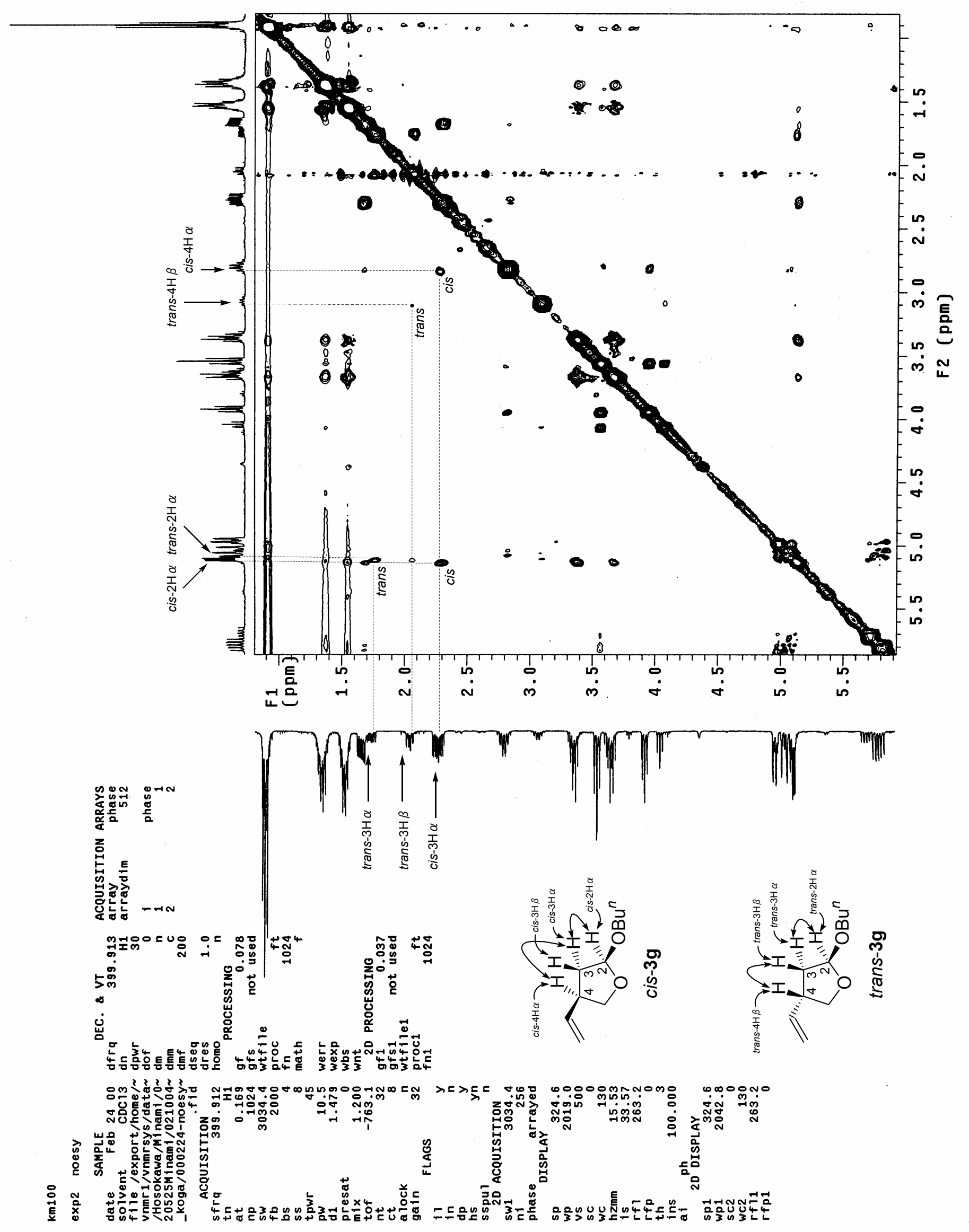

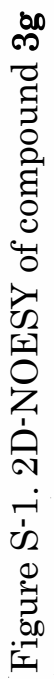




\section{$\mathrm{X}$-Ray Structure Analysis of $3 \mathrm{c}$ and $3 \mathrm{e}$.}

$\mathrm{X}$-ray analysis were performed on Rigaku AFC7R diffractometer with graphite monochromated Mo-Ka radiation $(\lambda=0.71069 \AA)$ and $12 \mathrm{~kW}$ rotating anode generator. Cell constants and orientation matrixes for data collection were obtained from least-square refinements using the setting angles of 20 carefully centered reflections corresponding to each cell system and dimensions. All calculations were performed using the teXsan crystallographic software package of Molecular Structure Corporation. The structures were solved by direct method (SIR88 or SIR92) and expanded using Fourier techniques. Neutral atom scattering factors were taken from Cromer and Waber. Anomalous dispersion effects were included in $F$ calc; the values for $\Delta f^{\prime \prime}$ and $\Delta f$ " were those of Creagh and McAuley. The values for the mass attenuation coefficients are those of Creagh and Hubbel. Non-hydrogen atoms were refined anisotropically. Hydrogen atoms were included in the calculation with isotropic temperature parameter on calculated positions riding on their carrier atoms.

Preparation of 3c was described previously on page S4. Single crystrals of 3c was obtained by recrystallization from hexane and EtOAc (5:1).

Preparation of $\left(R^{*} R^{*} 3 \mathrm{e}\right)$ was described previously on page S6. Single crystrals of $R * R * 3$ e was obtained by recrystallization from hexane and diethyl ether (3:1).

Table S-1. Crystal data and structure refinement for 3c.
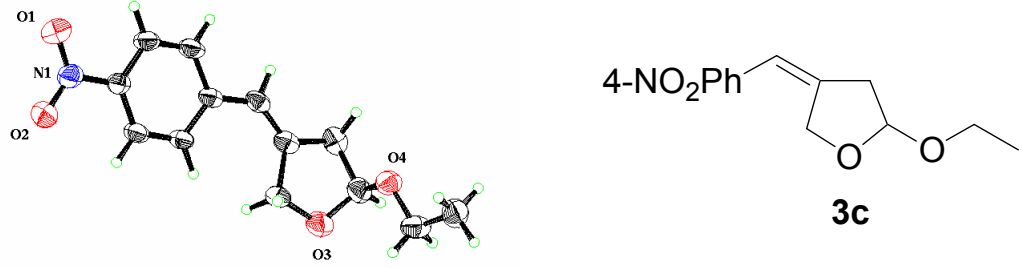

Figure S-2. ORTEP drawing of compound 3c.

\section{A. Crystal Data}

Empirical Formula

$\mathrm{C}_{13} \mathrm{H}_{15} \mathrm{NO}_{4}$

Formula Weight

249.27 
Crystal Color, Habit

Crystal Dimensions

Crystal System

Lattice Type

No. of Reflections Used for Unit

Cell Determination (2 $\theta$ range)

Omega Scan Peak Width

at Half-height

Lattice Parameters

Volume

Space Group

$\mathrm{Z}$ value

$\mathrm{D}_{\text {calc }}$

$\mathrm{F}(000)$

$\mu(\mathrm{MoKa})$

B. Intensity Measurements

Diffractometer

Radiation

Attenuator

Temperature

Voltage, Current

Collimator Size

Take-off Angle

Detector Aperture

Crystal to Detector Distance

Scan Type

Scan Rate

Scan Width

$2 \theta_{\max }$

No. of Reflections Measured yellow, plate

$0.30 \times 0.26 \times 0.17 \mathrm{~mm}^{3}$

triclinic

Primitive

$25\left(25.9-28.6^{\circ}\right)$

$0.33^{\circ}$

$\mathrm{a}=11.014(1) \AA \quad \alpha=95.35(1)^{\circ}$

$\mathrm{b}=13.147(2) \AA \quad \beta=97.18(1)^{\circ}$

$\mathrm{c}=4.8355(6) \AA \quad \gamma=113.337(8)^{\circ}$

$630.0(1) \AA^{3}$

P-1 (\#2)

2

$1.314 \mathrm{~g} / \mathrm{cm}^{3}$

264.00

$0.98 \mathrm{~cm}^{-1}$

Rigaku AFC7R (rotating anode)

$\operatorname{MoKa}(\lambda=0.71069 \AA)$

graphite monochromated

$\mathrm{Zr}$ foil (factor $=7.40)$

$23.0{ }^{\circ} \mathrm{C}$

$50 \mathrm{kV}, 250 \mathrm{~mA}$

$0.5 \mathrm{~mm}$

$6.0^{\circ}$

$3.0 \mathrm{~mm}$ horizontal

$4.0 \mathrm{~mm}$ vertical

$235 \mathrm{~mm}$

$\omega-2 \theta$

$4.0 \% \min$ (in $\omega$ ) (up to 5 scans)

$(1.84+0.30 \tan \theta)^{\circ}$

$55.0^{\circ}$

Total: 3042 
Corrections

C. Structure Solution and Refinement

Structure Solution

Refinement

Function Minimized

Least Squares Weights

p-factor

Anomalous Dispersion

No. of Reflections (All, $2 \sigma<55.00^{\circ}$ )

No. Variables

Reflection/Parameter Ratio

Residuals: R; $\mathrm{Rw}$

Residuals: R1

No. of Reflections to calc R1

Goodness of Fit Indicator

Max Shift/Error in Final Cycle

Maximum peak in Final Diff. Map

Minimum peak in Final Diff. Map

Table S-2. Atomic coordinates and $\mathrm{B}_{\mathrm{iso}} / \mathrm{B}_{\mathrm{eq}}$

$\begin{array}{llccl}\text { atom } & \mathrm{x} & \mathrm{y} & \mathrm{z} & \mathrm{B}_{\mathrm{eq}} \\ \mathrm{O}(1) & 0.6603(2) & 0.5439(2) & -0.1830(5) & 8.16(7) \\ \mathrm{O}(2) & 0.8724(2) & 0.5839(2) & -0.1017(5) & 7.74(7) \\ \mathrm{O}(3) & 0.9013(2) & 0.0825(2) & 0.8647(5) & 7.83(6) \\ \mathrm{O}(4) & 0.7393(2) & -0.0887(2) & 0.6318(4) & 6.18(5) \\ \mathrm{N}(1) & 0.7563(3) & 0.5281(2) & -0.0719(5) & 5.91(6) \\ \mathrm{C}(1) & 0.7317(2) & 0.4413(2) & 0.1062(5) & 4.83(6)\end{array}$

Unique: $2891\left(\mathrm{R}_{\mathrm{int}}=0.007\right)$

Lorentz-polarization

Absorption

(trans. factors: $0.9064-0.9982$ )

Secondary Extinction

(coefficient: 3.71810e-07)

Direct Methods (SIR88)

Full-matrix least-squares

$\Sigma \mathrm{w}\left(\mathrm{Fo}^{2}-\mathrm{Fc}^{2}\right)^{2}$

$1 / \mathrm{\sigma}^{2}\left(\mathrm{Fo}^{2}\right)$

0.0700

All non-hydrogen atoms

2891

164

17.63

$0.073 ; 0.206$

0.061

1512

1.50

0.001

$0.64 \mathrm{e}^{-} / \AA^{3}$

$-0.29 \mathrm{e}^{-} / \AA^{3}$ 


$\begin{array}{lllll}\mathrm{C}(2) & 0.6022(3) & 0.3813(3) & 0.1516(7) & 5.84(7) \\ \mathrm{C}(3) & 0.5805(2) & 0.2991(3) & 0.3152(6) & 5.70(7) \\ \mathrm{C}(4) & 0.6840(2) & 0.2727(2) & 0.4406(5) & 4.65(5) \\ \mathrm{C}(5) & 0.8135(2) & 0.3362(2) & 0.3886(6) & 5.46(6) \\ \mathrm{C}(6) & 0.8367(2) & 0.4185(2) & 0.2244(6) & 5.49(6) \\ \mathrm{C}(7) & 0.6513(2) & 0.1841(2) & 0.6099(6) & 5.11(6) \\ \mathrm{C}(8) & 0.7265(3) & 0.1347(2) & 0.7265(5) & 5.08(6) \\ \mathrm{C}(9) & 0.8703(3) & 0.1586(2) & 0.7116(6) & 5.77(7) \\ \mathrm{C}(10) & 0.6787(3) & 0.0404(2) & 0.8933(6) & 5.92(7) \\ \mathrm{C}(11) & 0.7816(3) & -0.0072(3) & 0.8871(7) & 6.48(8) \\ \mathrm{C}(12) & 0.8257(3) & -0.1437(3) & 0.5962(7) & 6.98(9) \\ \mathrm{C}(13) & 0.7585(4) & -0.2438(3) & 0.3776(8) & 7.68(10) \\ \mathrm{H}(1) & 0.5289 & 0.3976 & 0.0677 & 7.0 \\ \mathrm{H}(2) & 0.4911 & 0.2593 & 0.3524 & 6.9 \\ \mathrm{H}(3) & 0.8874 & 0.3209 & 0.4729 & 6.5 \\ \mathrm{H}(4) & 0.9260 & 0.4613 & 0.1941 & 6.6 \\ \mathrm{H}(5) & 0.5605 & 0.1558 & 0.6464 & 6.0 \\ \mathrm{H}(6) & 0.9291 & 0.2342 & 0.7916 & 7.0\end{array}$

Table S-3. Atomic coordinates and $\mathrm{B}_{\mathrm{iso}} / \mathrm{B}_{\mathrm{eq}}$ (continued)

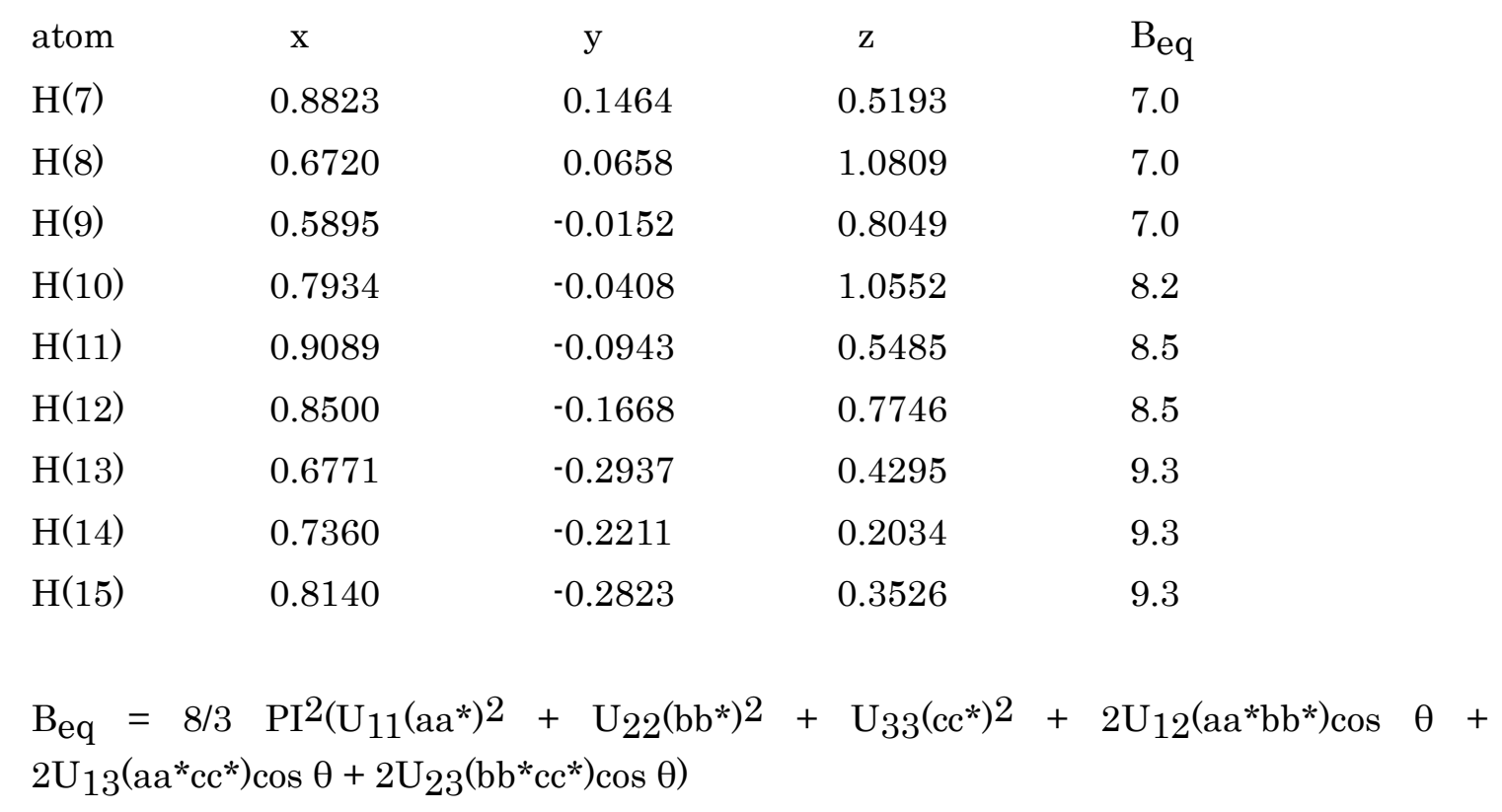


Table S-4. Anisotropic Displacement Parameters

$\begin{array}{lcccccc}\text { atom } & \mathrm{U}_{11} & \mathrm{U}_{22} & \mathrm{U}_{33} & \mathrm{U}_{12} & \mathrm{U}_{13} & \mathrm{U}_{23} \\ \mathrm{O}(1) & 0.086(2) & 0.108(2) & 0.126(2) & 0.051(1) & 0.005(1) & 0.024(2) \\ \mathrm{O}(2) & 0.075(1) & 0.081(1) & 0.140(2) & 0.030(1) & 0.030(1) & 0.025(1) \\ \mathrm{O}(3) & 0.071(1) & 0.095(2) & 0.111(2) & 0.021(1) & -0.017(1) & 0.021(1) \\ \mathrm{O}(4) & 0.071(1) & 0.078(1) & 0.078(1) & 0.0309(10) & -0.0039(9) & -0.0054(10) \\ \mathrm{N}(1) & 0.069(2) & 0.068(1) & 0.088(2) & 0.035(1) & 0.007(1) & -0.006(1) \\ \mathrm{C}(1) & 0.052(1) & 0.057(1) & 0.070(2) & 0.023(1) & 0.006(1) & -0.008(1) \\ \mathrm{C}(2) & 0.048(1) & 0.079(2) & 0.097(2) & 0.032(1) & 0.008(1) & 0.001(2) \\ \mathrm{C}(3) & 0.042(1) & 0.078(2) & 0.092(2) & 0.023(1) & 0.014(1) & -0.002(2) \\ \mathrm{C}(4) & 0.043(1) & 0.064(2) & 0.062(1) & 0.019(1) & 0.0072(10) & -0.011(1) \\ \mathrm{C}(5) & 0.042(1) & 0.073(2) & 0.087(2) & 0.021(1) & 0.007(1) & 0.007(2) \\ \mathrm{C}(6) & 0.042(1) & 0.069(2) & 0.090(2) & 0.018(1) & 0.009(1) & 0.001(2) \\ \mathrm{C}(7) & 0.047(1) & 0.065(2) & 0.071(2) & 0.014(1) & 0.018(1) & -0.011(1) \\ \mathrm{C}(8) & 0.055(1) & 0.072(2) & 0.054(1) & 0.019(1) & 0.007(1) & -0.011(1) \\ \mathrm{C}(9) & 0.052(1) & 0.075(2) & 0.081(2) & 0.020(1) & -0.003(1) & 0.005(1) \\ \mathrm{C}(10) & 0.084(2) & 0.071(2) & 0.062(2) & 0.022(1) & 0.024(1) & -0.001(1) \\ \mathrm{C}(11) & 0.074(2) & 0.072(2) & 0.085(2) & 0.019(2) & -0.004(1) & 0.012(2) \\ \mathrm{C}(12) & 0.065(2) & 0.108(3) & 0.096(2) & 0.041(2) & 0.013(2) & 0.006(2) \\ \mathrm{C}(13) & 0.096(2) & 0.082(2) & 0.122(3) & 0.039(2) & 0.041(2) & 0.010(2)\end{array}$

The general temperature factor expression:

$\exp \left(-2 \mathrm{PI} 2\left(\mathrm{a} * 2 \mathrm{U}_{11} \mathrm{~h}^{2}+\mathrm{b}^{* 2} \mathrm{U}_{22} \mathrm{k}^{2}+\mathrm{c}^{* 2} \mathrm{U}_{33} \mathrm{l}^{2}+2 \mathrm{a} * \mathrm{~b}^{*} \mathrm{U}_{12} \mathrm{hk}+2 \mathrm{a}{ }^{*} \mathrm{U}_{13} \mathrm{hl}+\right.\right.$ $\left.\left.2 \mathrm{~b} * \mathrm{c} * \mathrm{U}_{23} \mathrm{kl}\right)\right)$

Table S-5. Bond Lengths $(\AA)$

$\begin{array}{llllll}\text { atom } & \text { atom } & \text { distance } & \text { atom } & \text { atom } & \text { distance } \\ \mathrm{O}(1) & \mathrm{N}(1) & 1.226(3) & \mathrm{O}(2) & \mathrm{N}(1) & 1.232(3) \\ \mathrm{O}(3) & \mathrm{C}(9) & 1.418(4) & \mathrm{O}(3) & \mathrm{C}(11) & 1.403(4) \\ \mathrm{O}(4) & \mathrm{C}(11) & 1.451(4) & \mathrm{O}(4) & \mathrm{C}(12) & 1.422(4) \\ \mathrm{N}(1) & \mathrm{C}(1) & 1.454(4) & \mathrm{C}(1) & \mathrm{C}(2) & 1.387(4) \\ \mathrm{C}(1) & \mathrm{C}(6) & 1.377(3) & \mathrm{C}(2) & \mathrm{C}(3) & 1.361(4) \\ \mathrm{C}(2) & \mathrm{H}(1) & 0.97 & \mathrm{C}(3) & \mathrm{C}(4) & 1.403(4)\end{array}$




$\begin{array}{llllll}\mathrm{C}(3) & \mathrm{H}(2) & 0.96 & \mathrm{C}(4) & \mathrm{C}(5) & 1.407(3) \\ \mathrm{C}(4) & \mathrm{C}(7) & 1.441(4) & \mathrm{C}(5) & \mathrm{C}(6) & 1.363(4) \\ \mathrm{C}(5) & \mathrm{H}(3) & 0.97 & \mathrm{C}(6) & \mathrm{H}(4) & 0.96 \\ \mathrm{C}(7) & \mathrm{C}(8) & 1.343(4) & \mathrm{C}(7) & \mathrm{H}(5) & 0.97 \\ \mathrm{C}(8) & \mathrm{C}(9) & 1.500(4) & \mathrm{C}(8) & \mathrm{C}(10) & 1.497(4) \\ \mathrm{C}(9) & \mathrm{H}(6) & 0.96 & \mathrm{C}(9) & \mathrm{H}(7) & 0.96 \\ \mathrm{C}(10) & \mathrm{C}(11) & 1.498(4) & \mathrm{C}(10) & \mathrm{H}(8) & 0.96 \\ \mathrm{C}(10) & \mathrm{H}(9) & 0.97 & \mathrm{C}(11) & \mathrm{H}(10) & 0.98 \\ \mathrm{C}(12) & \mathrm{C}(13) & 1.473(5) & \mathrm{C}(12) & \mathrm{H}(11) & 0.96 \\ \mathrm{C}(12) & \mathrm{H}(12) & 0.98 & \mathrm{C}(13) & \mathrm{H}(13) & 0.96 \\ \mathrm{C}(13) & \mathrm{H}(14) & 0.96 & \mathrm{C}(13) & \mathrm{H}(15) & 0.95\end{array}$

Table S-6. Bond Angles $\left(^{\circ}\right)$

$\begin{array}{llllllll}\text { atom } & \text { atom } & \text { atom } & \text { angle } & \text { atom } & \text { atom } & \text { atom } & \text { angle } \\ & & & & & & \\ \mathrm{C}(9) & \mathrm{O}(3) & \mathrm{C}(11) & 109.3(2) & \mathrm{C}(11) & \mathrm{O}(4) & \mathrm{C}(12) & 114.4(2) \\ \mathrm{O}(1) & \mathrm{N}(1) & \mathrm{O}(2) & 122.6(3) & \mathrm{O}(1) & \mathrm{N}(1) & \mathrm{C}(1) & 118.4(2) \\ \mathrm{O}(2) & \mathrm{N}(1) & \mathrm{C}(1) & 119.0(2) & \mathrm{N}(1) & \mathrm{C}(1) & \mathrm{C}(2) & 119.5(2) \\ \mathrm{N}(1) & \mathrm{C}(1) & \mathrm{C}(6) & 119.7(2) & \mathrm{C}(2) & \mathrm{C}(1) & \mathrm{C}(6) & 120.8(3) \\ \mathrm{C}(1) & \mathrm{C}(2) & \mathrm{C}(3) & 118.9(2) & \mathrm{C}(1) & \mathrm{C}(2) & \mathrm{H}(1) & 120.4 \\ \mathrm{C}(3) & \mathrm{C}(2) & \mathrm{H}(1) & 120.8 & \mathrm{C}(2) & \mathrm{C}(3) & \mathrm{C}(4) & 122.6(2) \\ \mathrm{C}(2) & \mathrm{C}(3) & \mathrm{H}(2) & 118.8 & \mathrm{C}(4) & \mathrm{C}(3) & \mathrm{H}(2) & 118.6 \\ \mathrm{C}(3) & \mathrm{C}(4) & \mathrm{C}(5) & 116.3(3) & \mathrm{C}(3) & \mathrm{C}(4) & \mathrm{C}(7) & 118.7(2) \\ \mathrm{C}(5) & \mathrm{C}(4) & \mathrm{C}(7) & 125.0(2) & \mathrm{C}(4) & \mathrm{C}(5) & \mathrm{C}(6) & 121.7(2) \\ \mathrm{C}(4) & \mathrm{C}(5) & \mathrm{H}(3) & 118.7 & \mathrm{C}(6) & \mathrm{C}(5) & \mathrm{H}(3) & 119.6 \\ \mathrm{C}(1) & \mathrm{C}(6) & \mathrm{C}(5) & 119.8(2) & \mathrm{C}(1) & \mathrm{C}(6) & \mathrm{H}(4) & 120.1 \\ \mathrm{C}(5) & \mathrm{C}(6) & \mathrm{H}(4) & 120.1 & \mathrm{C}(4) & \mathrm{C}(7) & \mathrm{C}(8) & 130.7(2) \\ \mathrm{C}(4) & \mathrm{C}(7) & \mathrm{H}(5) & 114.7 & \mathrm{C}(8) & \mathrm{C}(7) & \mathrm{H}(5) & 114.6 \\ \mathrm{C}(7) & \mathrm{C}(8) & \mathrm{C}(9) & 128.2(3) & \mathrm{C}(7) & \mathrm{C}(8) & \mathrm{C}(10) & 125.4(2) \\ \mathrm{C}(9) & \mathrm{C}(8) & \mathrm{C}(10) & 106.4(3) & \mathrm{O}(3) & \mathrm{C}(9) & \mathrm{C}(8) & 106.0(2) \\ \mathrm{O}(3) & \mathrm{C}(9) & \mathrm{H}(6) & 110.5 & \mathrm{O}(3) & \mathrm{C}(9) & \mathrm{H}(7) & 109.6 \\ \mathrm{C}(8) & \mathrm{C}(9) & \mathrm{H}(6) & 111.7 & \mathrm{C}(8) & \mathrm{C}(9) & \mathrm{H}(7) & 111.1 \\ \mathrm{H}(6) & \mathrm{C}(9) & \mathrm{H}(7) & 108.0 & \mathrm{C}(8) & \mathrm{C}(10) & \mathrm{C}(11) & 103.1(2) \\ \mathrm{C}(8) & \mathrm{C}(10) & \mathrm{H}(8) & 111.9 & \mathrm{C}(8) & \mathrm{C}(10) & \mathrm{H}(9) & 110.9 \\ \mathrm{C}(11) & \mathrm{C}(10) & \mathrm{H}(8) & 113.1 & \mathrm{C}(11) & \mathrm{C}(10) & \mathrm{H}(9) & 111.3\end{array}$




$\begin{array}{llllllll}\mathrm{H}(8) & \mathrm{C}(10) & \mathrm{H}(9) & 106.7 & \mathrm{O}(3) & \mathrm{C}(11) & \mathrm{O}(4) & 108.5(3) \\ \mathrm{O}(3) & \mathrm{C}(11) & \mathrm{C}(10) & 105.9(3) & \mathrm{O}(3) & \mathrm{C}(11) & \mathrm{H}(10) & 111.1 \\ \mathrm{O}(4) & \mathrm{C}(11) & \mathrm{C}(10) & 107.2(2) & \mathrm{O}(4) & \mathrm{C}(11) & \mathrm{H}(10) & 111.4 \\ \mathrm{C}(10) & \mathrm{C}(11) & \mathrm{H}(10) & 112.5 & \mathrm{O}(4) & \mathrm{C}(12) & \mathrm{C}(13) & 110.4(3)\end{array}$

Table S-7. Bond Angles $\left(^{\circ}\right) \quad$ (continued)

$\begin{array}{llllllll}\text { atom } & \text { atom } & \text { atom } & \text { angle } & \text { atom } & \text { atom } & \text { atom } & \text { angle } \\ & & & & & & & \\ \mathrm{O}(4) & \mathrm{C}(12) & \mathrm{H}(11) & 111.4 & \mathrm{O}(4) & \mathrm{C}(12) & \mathrm{H}(12) & 109.8 \\ \mathrm{C}(13) & \mathrm{C}(12) & \mathrm{H}(11) & 110.1 & \mathrm{C}(13) & \mathrm{C}(12) & \mathrm{H}(12) & 109.0 \\ \mathrm{H}(11) & \mathrm{C}(12) & \mathrm{H}(12) & 106.0 & \mathrm{C}(12) & \mathrm{C}(13) & \mathrm{H}(13) & 109.5 \\ \mathrm{C}(12) & \mathrm{C}(13) & \mathrm{H}(14) & 108.9 & \mathrm{C}(12) & \mathrm{C}(13) & \mathrm{H}(15) & 111.7 \\ \mathrm{H}(13) & \mathrm{C}(13) & \mathrm{H}(14) & 108.4 & \mathrm{H}(13) & \mathrm{C}(13) & \mathrm{H}(15) & 109.0 \\ \mathrm{H}(14) & \mathrm{C}(13) & \mathrm{H}(15) & 109.2 & & & & \end{array}$

Table S-8. Torsion Angles $\left(^{\circ}\right)$

$\begin{array}{lllllllllr}\text { atom } & \text { atom } & \text { atom } & \text { atom } & \text { angle } & \text { atom } & \text { atom } & \text { atom } & \text { atom } & \text { angle } \\ \mathrm{O}(1) & \mathrm{N}(1) & \mathrm{C}(1) & \mathrm{C}(2) & -1.7(4) & \mathrm{O}(1) & \mathrm{N}(1) & \mathrm{C}(1) & \mathrm{C}(6) & 177.3(2) \\ \mathrm{O}(2) & \mathrm{N}(1) & \mathrm{C}(1) & \mathrm{C}(2) & 177.2(2) & \mathrm{O}(2) & \mathrm{N}(1) & \mathrm{C}(1) & \mathrm{C}(6) & -3.8(4) \\ \mathrm{O}(3) & \mathrm{C}(9) & \mathrm{C}(8) & \mathrm{C}(7) & -179.0(2) & \mathrm{O}(3) & \mathrm{C}(9) & \mathrm{C}(8) & \mathrm{C}(10) & -0.6(3) \\ \mathrm{O}(3) & \mathrm{C}(11) & \mathrm{O}(4) & \mathrm{C}(12) & 67.2(3) & \mathrm{O}(3) & \mathrm{C}(11) & \mathrm{C}(10) & \mathrm{C}(8) & 28.9(3) \\ \mathrm{O}(4) & \mathrm{C}(11) & \mathrm{O}(3) & \mathrm{C}(9) & 83.7(3) & \mathrm{O}(4) & \mathrm{C}(11) & \mathrm{C}(10) & \mathrm{C}(8) & -86.7(3) \\ \mathrm{N}(1) & \mathrm{C}(1) & \mathrm{C}(2) & \mathrm{C}(3) & 179.0(2) & \mathrm{N}(1) & \mathrm{C}(1) & \mathrm{C}(6) & \mathrm{C}(5) & -179.2(2) \\ \mathrm{C}(1) & \mathrm{C}(2) & \mathrm{C}(3) & \mathrm{C}(4) & 0.1(4) & \mathrm{C}(1) & \mathrm{C}(6) & \mathrm{C}(5) & \mathrm{C}(4) & 0.3(4) \\ \mathrm{C}(2) & \mathrm{C}(1) & \mathrm{C}(6) & \mathrm{C}(5) & -0.2(4) & \mathrm{C}(2) & \mathrm{C}(3) & \mathrm{C}(4) & \mathrm{C}(5) & 0.0(4) \\ \mathrm{C}(2) & \mathrm{C}(3) & \mathrm{C}(4) & \mathrm{C}(7) & -179.8(3) & \mathrm{C}(3) & \mathrm{C}(2) & \mathrm{C}(1) & \mathrm{C}(6) & 0.0(4) \\ \mathrm{C}(3) & \mathrm{C}(4) & \mathrm{C}(5) & \mathrm{C}(6) & -0.2(4) & \mathrm{C}(3) & \mathrm{C}(4) & \mathrm{C}(7) & \mathrm{C}(8) & 172.0(2) \\ \mathrm{C}(4) & \mathrm{C}(7) & \mathrm{C}(8) & \mathrm{C}(9) & -0.2(4) & \mathrm{C}(4) & \mathrm{C}(7) & \mathrm{C}(8) & \mathrm{C}(10) & -178.3(2) \\ \mathrm{C}(5) & \mathrm{C}(4) & \mathrm{C}(7) & \mathrm{C}(8) & -7.8(4) & \mathrm{C}(6) & \mathrm{C}(5) & \mathrm{C}(4) & \mathrm{C}(7) & 179.6(3) \\ \mathrm{C}(7) & \mathrm{C}(8) & \mathrm{C}(10) & \mathrm{C}(11) & 161.6(2) & \mathrm{C}(8) & \mathrm{C}(9) & \mathrm{O}(3) & \mathrm{C}(11) & 19.7(3) \\ \mathrm{C}(9) & \mathrm{O}(3) & \mathrm{C}(11) & \mathrm{C}(10) & -31.0(3) & \mathrm{C}(9) & \mathrm{C}(8) & \mathrm{C}(10) & \mathrm{C}(11) & -16.9(3) \\ \mathrm{C}(10) & \mathrm{C}(11) & \mathrm{O}(4) & \mathrm{C}(12) & -178.8(2) & \mathrm{C}(11) & \mathrm{O}(4) & \mathrm{C}(12) & \mathrm{C}(13) & 165.1(3)\end{array}$


Table S-9. Non-bonded Contacts out to $3.60 \AA$

\begin{tabular}{|c|c|c|c|c|c|}
\hline atom & atom & distance & atom & atom & distance \\
\hline $\mathrm{O}(1)$ & $\mathrm{H}(1)^{1)}$ & 2.59 & $\mathrm{O}(1)$ & $\mathrm{H}(13)^{2)}$ & 2.94 \\
\hline $\mathrm{O}(1)$ & $\mathrm{H}(15)^{3)}$ & 3.09 & $\mathrm{O}(1)$ & $\mathrm{H}(14)^{3)}$ & 3.20 \\
\hline $\mathrm{O}(1)$ & $\mathrm{C}(2)^{1)}$ & $3.419(3)$ & $\mathrm{O}(1)$ & $\mathrm{H}(13)^{3)}$ & 3.43 \\
\hline $\mathrm{O}(1)$ & $\mathrm{C}(13)^{3)}$ & $3.431(4)$ & $\mathrm{O}(1)$ & $\mathrm{H}(15)^{2)}$ & 3.46 \\
\hline $\mathrm{O}(1)$ & $\mathrm{C}(2)^{4)}$ & $3.529(4)$ & $\mathrm{O}(1)$ & $\mathrm{C}(13)^{2)}$ & $3.573(5)$ \\
\hline $\mathrm{O}(1)$ & $\mathrm{C}(3)^{4)}$ & $3.575(4)$ & $\mathrm{O}(1)$ & $\mathrm{H}(12)^{2)}$ & 3.58 \\
\hline $\mathrm{O}(2)$ & $H(4)^{5)}$ & 2.59 & $\mathrm{O}(2)$ & $H(6)^{6)}$ & 2.67 \\
\hline $\mathrm{O}(2)$ & $\mathrm{H}(15)^{3)}$ & 2.98 & $\mathrm{O}(2)$ & $\mathrm{H}(3)^{5)}$ & 3.28 \\
\hline $\mathrm{O}(2)$ & $\mathrm{C}(6)^{5)}$ & $3.342(3)$ & $\mathrm{O}(2)$ & $\mathrm{H}(15)^{2)}$ & 3.44 \\
\hline $\mathrm{O}(2)$ & $\mathrm{H}(12)^{2)}$ & 3.48 & $\mathrm{O}(2)$ & $\mathrm{H}(3)^{6)}$ & 3.53 \\
\hline $\mathrm{O}(2)$ & $\mathrm{C}(9)^{6)}$ & $3.595(4)$ & $\mathrm{O}(3)$ & $\mathrm{H}(12)^{7)}$ & 2.80 \\
\hline $\mathrm{O}(3)$ & $\mathrm{H}(11)^{8)}$ & 3.04 & $\mathrm{O}(3)$ & $\mathrm{H}(7)^{9)}$ & 3.25 \\
\hline $\mathrm{O}(3)$ & $\mathrm{H}(11)^{7)}$ & 3.26 & $\mathrm{O}(3)$ & $\mathrm{C}(12)^{7)}$ & $3.495(4)$ \\
\hline $\mathrm{O}(3)$ & $H(15)^{8)}$ & 3.56 & $\mathrm{O}(3)$ & $\mathrm{H}(10)^{7)}$ & 3.60 \\
\hline $\mathrm{O}(4)$ & $\mathrm{H}(2)^{10)}$ & 2.66 & $\mathrm{O}(4)$ & $\mathrm{H}(10)^{4)}$ & 2.99 \\
\hline $\mathrm{O}(4)$ & $\mathrm{H}(5)^{10)}$ & 3.14 & $\mathrm{O}(4)$ & $\mathrm{H}(14)^{9)}$ & 3.40 \\
\hline $\mathrm{O}(4)$ & $\mathrm{C}(3)^{10)}$ & $3.573(3)$ & $\mathrm{N}(1)$ & $\mathrm{H}(15)^{3)}$ & 2.88 \\
\hline $\mathrm{N}(1)$ & $\mathrm{C}(13)^{3)}$ & $3.526(4)$ & $\mathrm{N}(1)$ & $\mathrm{H}(14)^{3)}$ & 3.54 \\
\hline $\mathrm{N}(1)$ & $\mathrm{H}(4)^{5)}$ & 3.57 & $\mathrm{C}(1)$ & $\mathrm{H}(15)^{3)}$ & 3.43 \\
\hline $\mathrm{C}(1)$ & $\mathrm{C}(4)^{4)}$ & $3.588(3)$ & $\mathrm{C}(2)$ & $\mathrm{H}(5)^{4)}$ & 3.50 \\
\hline $\mathrm{C}(2)$ & $\mathrm{H}(14)^{11)}$ & 3.57 & $\mathrm{C}(3)$ & $\mathrm{H}(13)^{10)}$ & 3.21 \\
\hline $\mathrm{C}(3)$ & $\mathrm{H}(9)^{10)}$ & 3.40 & $\mathrm{C}(3)$ & $\mathrm{H}(5)^{4)}$ & 3.52 \\
\hline $\mathrm{C}(4)$ & $\mathrm{H}(8)^{4)}$ & 3.04 & $\mathrm{C}(4)$ & $\mathrm{H}(9)^{10)}$ & 3.50 \\
\hline $\mathrm{C}(5)$ & $\mathrm{H}(4)^{6)}$ & 3.30 & $\mathrm{C}(5)$ & $H(8)^{4)}$ & 3.37 \\
\hline
\end{tabular}

Table S-10. Non-bonded Contacts out to $3.60 \AA$ (continued)

$\begin{array}{llllll}\text { atom } & \text { atom } & \text { distance } & \text { atom } & \text { atom } & \text { distance } \\ & & & & & \\ \mathrm{C}(6) & \mathrm{H}(4)^{6)} & 3.36 & \mathrm{C}(6) & \mathrm{H}(4)^{5)} & 3.47 \\ \mathrm{C}(6) & \mathrm{H}(3)^{6)} & 3.58 & \mathrm{C}(6) & \mathrm{H}(6)^{4)} & 3.58 \\ \mathrm{C}(7) & \mathrm{H}(8)^{4)} & 2.95 & \mathrm{C}(7) & \mathrm{H}(9)^{10)} & 3.03\end{array}$




$\begin{array}{llllll}\mathrm{C}(8) & \mathrm{H}(8)^{4)} & 3.08 & \mathrm{C}(9) & \mathrm{H}(11)^{8)} & 3.23 \\ \mathrm{C}(9) & \mathrm{H}(15)^{8)} & 3.27 & \mathrm{C}(9) & \mathrm{H}(8)^{4)} & 3.33 \\ \mathrm{C}(10) & \mathrm{H}(7)^{9)} & 3.34 & \mathrm{C}(10) & \mathrm{H}(9)^{12)} & 3.37 \\ \mathrm{C}(10) & \mathrm{H}(5)^{10)} & 3.47 & \mathrm{C}(10) & \mathrm{H}(8)^{12)} & 3.57 \\ \mathrm{C}(11) & \mathrm{H}(14)^{9)} & 3.22 & \mathrm{C}(11) & \mathrm{H}(7)^{9)} & 3.30 \\ \mathrm{C}(11) & \mathrm{H}(2)^{10)} & 3.45 & \mathrm{C}(12) & \mathrm{H}(10)^{4)} & 3.10 \\ \mathrm{C}(12) & \mathrm{H}(2)^{10)} & 3.26 & \mathrm{C}(12) & \mathrm{H}(14)^{9)} & 3.33 \\ \mathrm{C}(12) & \mathrm{H}(7)^{8)} & 3.35 & \mathrm{C}(12) & \mathrm{H}(11)^{8)} & 3.54 \\ \mathrm{C}(13) & \mathrm{H}(2)^{10)} & 3.13 & \mathrm{C}(13) & \mathrm{H}(10)^{4)} & 3.13 \\ \mathrm{C}(13) & \mathrm{H}(12)^{4)} & 3.32 & \mathrm{C}(13) & \mathrm{H}(1)^{11)} & 3.34 \\ \mathrm{C}(13) & \mathrm{H}(6)^{8)} & 3.59 & \mathrm{C}(13) & \mathrm{H}(7)^{8)} & 3.59 \\ \mathrm{H}(1) & \mathrm{H}(13)^{11)} & 2.89 & \mathrm{H}(1) & \mathrm{H}(14)^{11)} & 2.95 \\ \mathrm{H}(1) & \mathrm{H}(1)^{1)} & 3.11 & \mathrm{H}(1) & \mathrm{H}(13)^{10)} & 3.53 \\ \mathrm{H}(2) & \mathrm{H}(13)^{10)} & 2.41 & \mathrm{H}(2) & \mathrm{H}(9)^{10)} & 2.96 \\ \mathrm{H}(2) & \mathrm{H}(14)^{11)} & 3.29 & \mathrm{H}(2) & \mathrm{H}(12)^{10)} & 3.41 \\ \mathrm{H}(2) & \mathrm{H}(14)^{10)} & 3.42 & \mathrm{H}(2) & \mathrm{H}(10)^{10)} & 3.50 \\ \mathrm{H}(3) & \mathrm{H}(4)^{6)} & 2.94 & \mathrm{H}(3) & \mathrm{H}(8)^{4)} & 3.44 \\ \mathrm{H}(3) & \mathrm{H}(6)^{4)} & 3.53 & \mathrm{H}(3) & \mathrm{H}(15)^{8)} & 3.53 \\ \mathrm{H}(4) & \mathrm{H}(4)^{5)} & 2.65 & \mathrm{H}(4)^{6)} & 3.06 \\ \mathrm{H}(4) & \mathrm{H}(6)^{4)} & 3.42 & \mathrm{H}(4) & \mathrm{H}(9)^{10} & 2.61 \\ \mathrm{H}(5) & \mathrm{H}(8)^{4)} & 3.43 & \mathrm{H}(5) & \mathrm{H}(8)^{12} & 3.53 \\ & & & & \end{array}$

Table S-11. Non-bonded Contacts out to $3.60 \AA$ (continued)

$\begin{array}{llllll}\text { atom } & \text { atom } & \text { distance } & \text { atom } & \text { atom } & \text { distance } \\ \mathrm{H}(5) & \mathrm{H}(9)^{12)} & 3.57 & \mathrm{H}(6) & \mathrm{H}(15)^{8)} & 2.84 \\ \mathrm{H}(6) & \mathrm{H}(12)^{7)} & 3.45 & \mathrm{H}(6) & \mathrm{H}(11)^{8)} & 3.47 \\ \mathrm{H}(7) & \mathrm{H}(11)^{8)} & 2.70 & \mathrm{H}(7) & \mathrm{H}(8)^{4)} & 2.72 \\ \mathrm{H}(7) & \mathrm{H}(10)^{4)} & 2.93 & \mathrm{H}(7) & \mathrm{H}(15)^{8)} & 3.05 \\ \mathrm{H}(7) & \mathrm{H}(12)^{8)} & 3.36 & \mathrm{H}(8) & \mathrm{H}(9)^{12)} & 2.83 \\ \mathrm{H}(8) & \mathrm{H}(8)^{12)} & 3.43 & \mathrm{H}(9) & \mathrm{H}(9)^{12)} & 3.00 \\ \mathrm{H}(9) & \mathrm{H}(9)^{10)} & 3.48 & \mathrm{H}(10) & \mathrm{H}(14)^{9)} & 2.40 \\ \mathrm{H}(10) & \mathrm{H}(11)^{9)} & 2.84 & \mathrm{H}(10) & \mathrm{H}(11)^{7)} & 3.28 \\ \mathrm{H}(11) & \mathrm{H}(11)^{8)} & 2.63 & \mathrm{H}(12) & \mathrm{H}(14)^{9)} & 2.57 \\ \mathrm{H}(12) & \mathrm{H}(15)^{9)} & 3.30 & & & \end{array}$


Symmetry operations
(1) $\quad-\mathrm{X}+1,-\mathrm{Y}+1,-\mathrm{Z}$
(2) $\quad \mathrm{X}, \mathrm{Y}+1, \mathrm{Z}-1$
(3) $\quad \mathrm{X}, \mathrm{Y}+1, \mathrm{Z}$
(4) $\quad \mathrm{X}, \mathrm{Y}, \mathrm{Z}-1$
(5) $\quad-\mathrm{X}+2,-\mathrm{Y}+1,-\mathrm{Z}$
(6) $\quad-\mathrm{X}+2,-\mathrm{Y}+1,-\mathrm{Z}+1$
(7) $\quad-\mathrm{X}+2,-\mathrm{Y},-\mathrm{Z}+2$
(8) $\quad-\mathrm{X}+2,-\mathrm{Y},-\mathrm{Z}+1$
(9) $\quad \mathrm{X}, \mathrm{Y}, \mathrm{Z}+1$
(10) $\quad-\mathrm{X}+1,-\mathrm{Y},-\mathrm{Z}+1$
(11) $\quad-\mathrm{X}+1,-\mathrm{Y},-\mathrm{Z}$
(12) $\quad-\mathrm{X}+1,-\mathrm{Y},-\mathrm{Z}+2$

Table S-12. Crystal data and structure refinement for $R^{*} R^{*} 3$ e.
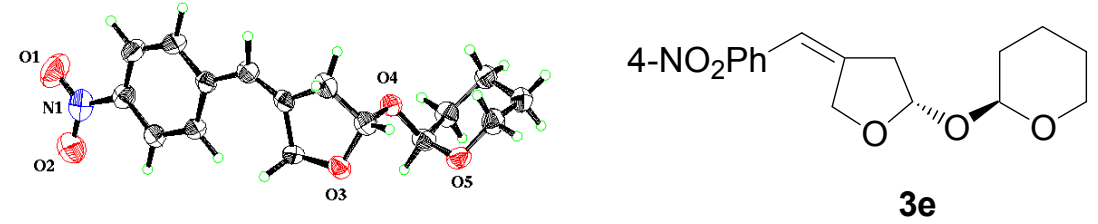

Figure S-3. ORTEP drawing of compound 3e.

\section{A. Crystal Data}

Empirical Formula

Formula Weight

Crystal Color, Habit

Crystal Dimensions

Crystal System

Lattice Type

No. of Reflections Used for Unit

Cell Determination (2 $\theta$ range)

Omega Scan Peak Width at Half-height

Lattice Parameters

Volume

Space Group

$\mathrm{Z}$ value
$\mathrm{C}_{16} \mathrm{H}_{19} \mathrm{NO}_{5}$ 305.33

pale_yellow, rectangular

0.25 X 0.15 X $0.15 \mathrm{~mm}$

monoclinic

C-centered

$25\left(22.1-23.8^{\circ}\right)$

$0.34^{\circ}$

$\mathrm{a}=19.238(4) \AA$

$\mathrm{b}=12.755(3) \AA \quad \beta=124.62(1)^{\circ}$

$\mathrm{c}=15.148(3) \AA$

3058(1) $\AA^{3}$

$\mathrm{C} 2 / \mathrm{c}(\# 15)$

8 
$\mathrm{D}_{\text {calc }}$

$\mathrm{F}(000)$

$\mathrm{m}(\mathrm{MoKa})$

B. Intensity Measurements

Diffractometer

Radiation

Attenuator

Temperature

Voltage, Current

Collimator Size

Take-off Angle

Detector Aperture

Crystal to Detector Distance

Scan Type

Scan Rate

Scan Width

$2 \theta_{\max }$

No. of Reflections Measured

Corrections

C. Structure Solution and Refinement

Structure Solution

Refinement

Function Minimized

Least Squares Weights

$\mathrm{p}$-factor

Anomalous Dispersion

No. of Reflections (All, $2 \theta<54.99^{\circ}$ )

No. Variables

Reflection/Parameter Ratio
$1.326 \mathrm{~g} / \mathrm{cm}^{3}$

1296.00

$0.99 \mathrm{~cm}^{-1}$
Rigaku AFC7R (rotating anode)

$\operatorname{MoKa}(\lambda=0.71069 \AA)$

graphite monochromated

Zr foil (factor $=7.40)$

$25.0{ }^{\circ} \mathrm{C}$

$50 \mathrm{kV}, 250 \mathrm{~mA}$

$0.5 \mathrm{~mm}$

$6.0^{\circ}$

$3.0 \mathrm{~mm}$ horizontal

$6.5 \mathrm{~mm}$ vertical

$235 \mathrm{~mm}$

$\omega-2 \theta$

$8.0^{\circ} / \mathrm{min}$ (in $\omega$ ) (up to 5 scans)

$(1.52+0.30 \tan \theta)^{\circ}$

$55.0^{\circ}$

Total: 3516

Unique: $3259\left(\mathrm{R}_{\mathrm{int}}=0.048\right)$

Lorentz-polarization

Direct Methods (SIR92)

Full-matrix least-squares

$\mathrm{S} \mathrm{w}\left(\mathrm{Fo}^{2}-\mathrm{Fc}^{2}\right)^{2}$

$1 / \mathrm{s}^{2}\left(\mathrm{Fo}^{2}\right)$

0.0350

All non-hydrogen atoms

3259

199

16.38 
Residuals: $\mathrm{R} ; \mathrm{Rw}$

Residuals: R1

No. of Reflections to calc R1

Goodness of Fit Indicator

Max Shift/Error in Final Cycle

Maximum peak in Final Diff. Map

Minimum peak in Final Diff. Map
$0.147 ; 0.163$

0.052

842

0.99

0.001

$0.69 \mathrm{e}^{-} / \AA^{3}$

$-0.70 \mathrm{e}^{-} / \AA^{3}$

Table S-13. Atomic coordinates and $\mathrm{B}_{\mathrm{iso}} / \mathrm{B}_{\mathrm{eq}}$

$\begin{array}{lllll}\text { atom } & \mathrm{x} & \mathrm{y} & \mathrm{z} & \mathrm{B} \text { eq } \\ \mathrm{O}(1) & 0.5013(3) & 0.8420(3) & 0.1224(4) & 10.7(1) \\ \mathrm{O}(2) & 0.6239(3) & 0.8177(3) & 0.1566(4) & 9.6(1) \\ \mathrm{O}(3) & 0.6824(2) & 0.1562(2) & 0.2181(2) & 5.34(8) \\ \mathrm{O}(4) & 0.6412(2) & 0.1065(2) & 0.3312(2) & 4.87(7) \\ \mathrm{O}(5) & 0.7465(2) & -0.0225(3) & 0.4049(2) & 5.76(9) \\ \mathrm{N}(1) & 0.5575(4) & 0.7838(4) & 0.1399(4) & 7.1(1) \\ \mathrm{C}(1) & 0.5268(3) & 0.4544(4) & 0.1369(4) & 4.82(10) \\ \mathrm{C}(2) & 0.4616(3) & 0.5226(4) & 0.1115(4) & 6.2(1) \\ \mathrm{C}(3) & 0.4711(3) & 0.6298(4) & 0.1129(4) & 6.3(1) \\ \mathrm{C}(4) & 0.5460(3) & 0.6712(4) & 0.1394(4) & 5.3(1) \\ \mathrm{C}(5) & 0.6127(3) & 0.6059(4) & 0.1656(4) & 5.7(1) \\ \mathrm{C}(6) & 0.6031(3) & 0.4986(4) & 0.1640(4) & 5.0(1) \\ \mathrm{C}(7) & 0.5117(3) & 0.3423(4) & 0.1330(4) & 5.0(1) \\ \mathrm{C}(8) & 0.6246(3) & 0.0970(4) & 0.2271(3) & 5.2(1) \\ \mathrm{C}(9) & 0.5397(3) & 0.1482(4) & 0.1490(4) & 5.6(1) \\ \mathrm{C}(10) & 0.5627(3) & 0.2612(4) & 0.1556(3) & 4.55(9) \\ \mathrm{C}(11) & 0.6549(3) & 0.2631(4) & 0.1976(4) & 4.84(10) \\ \mathrm{C}(12) & 0.7247(3) & 0.0789(4) & 0.4164(4) & 5.14(10) \\ \mathrm{C}(13) & 0.7306(3) & 0.0946(4) & 0.5195(3) & 5.6(1) \\ \mathrm{C}(14) & 0.6860(3) & 0.0079(4) & 0.5375(3) & 5.6(1) \\ \mathrm{C}(15) & 0.7132(3) & -0.0979(4) & 0.5227(4) & 6.2(1) \\ \mathrm{C}(16) & 0.6999(3) & -0.1030(4) & 0.4149(4) & 6.0(1) \\ \mathrm{H}(1) & 0.4041 & 0.4893 & 0.0927 & 8.5 \\ \mathrm{H}(2) & 0.4207 & 0.6772 & 0.0928 & 8.0 \\ & & & & \end{array}$


Table S-14. Atomic coordinates and $\mathrm{B}_{\mathrm{iso}} / \mathrm{B}_{\mathrm{eq}}$ (continued)

$\begin{array}{lllll}\text { atom } & \mathrm{x} & \mathrm{y} & \mathrm{z} & \mathrm{B} \text { eq } \\ \mathrm{H}(3) & 0.6671 & 0.6368 & 0.1849 & 7.3 \\ \mathrm{H}(4) & 0.6513 & 0.4496 & 0.1819 & 7.0 \\ \mathrm{H}(5) & 0.4507 & 0.3243 & 0.1049 & 6.9 \\ \mathrm{H}(6) & 0.6260 & 0.0205 & 0.2080 & 7.6 \\ \mathrm{H}(7) & 0.5100 & 0.1203 & 0.0756 & 7.2 \\ \mathrm{H}(8) & 0.5002 & 0.1355 & 0.1696 & 7.2 \\ \mathrm{H}(9) & 0.6886 & 0.3115 & 0.2613 & 7.4 \\ \mathrm{H}(10) & 0.6641 & 0.3001 & 0.1449 & 7.4 \\ \mathrm{H}(11) & 0.7675 & 0.1316 & 0.4202 & 7.4 \\ \mathrm{H}(12) & 0.7899 & 0.0994 & 0.5835 & 6.8 \\ \mathrm{H}(13) & 0.7062 & 0.1625 & 0.5192 & 6.8 \\ \mathrm{H}(14) & 0.6987 & 0.0127 & 0.6086 & 5.9 \\ \mathrm{H}(15) & 0.6276 & 0.0154 & 0.4868 & 5.9 \\ \mathrm{H}(16) & 0.7725 & -0.1098 & 0.5795 & 6.9 \\ \mathrm{H}(17) & 0.6822 & -0.1527 & 0.5310 & 6.9 \\ \mathrm{H}(18) & 0.6409 & -0.0984 & 0.3607 & 7.2 \\ \mathrm{H}(19) & 0.7183 & -0.1721 & 0.4073 & 7.2\end{array}$

$\mathrm{B}_{\mathrm{eq}}=8 / 3 \mathrm{PI}^{2}\left(\mathrm{U}_{11}\left(\mathrm{aa}^{*}\right)^{2}+\mathrm{U}_{22}\left(\mathrm{bb}^{*}\right)^{2}+\mathrm{U}_{33}\left(\mathrm{cc}^{*}\right)^{2}+2 \mathrm{U}_{12}\left(\mathrm{aa}^{*} \mathrm{bb}^{*}\right) \cos \theta+\right.$ $\left.2 \mathrm{U}_{13}(\mathrm{aa} * \mathrm{cc} *) \cos \theta+2 \mathrm{U}_{23}\left(\mathrm{bb}^{*} \mathrm{cc} *\right) \cos \theta\right)$

Table S-15. Anisotropic Displacement Parameters

$\begin{array}{lcccccc}\text { atom } & \mathrm{U}_{11} & \mathrm{U}_{22} & \mathrm{U}_{33} & \mathrm{U}_{12} & \mathrm{U}_{13} & \mathrm{U}_{23} \\ \mathrm{O}(1) & 0.135(4) & 0.087(3) & 0.177(4) & 0.042(3) & 0.083(4) & 0.014(3) \\ \mathrm{O}(2) & 0.129(4) & 0.089(3) & 0.157(4) & -0.013(3) & 0.088(4) & 0.009(3) \\ \mathrm{O}(3) & 0.082(2) & 0.068(2) & 0.072(2) & -0.002(2) & 0.054(2) & 0.002(2) \\ \mathrm{O}(4) & 0.061(2) & 0.072(2) & 0.053(2) & 0.003(2) & 0.033(1) & 0.005(2) \\ \mathrm{O}(5) & 0.066(2) & 0.089(2) & 0.072(2) & 0.015(2) & 0.044(2) & 0.005(2) \\ \mathrm{N}(1) & 0.115(4) & 0.072(3) & 0.068(3) & 0.006(2) & 0.043(4) & -0.004(3) \\ \mathrm{C}(1) & 0.051(2) & 0.080(3) & 0.044(3) & -0.001(2) & 0.022(3) & 0.011(3) \\ \mathrm{C}(2) & 0.047(3) & 0.096(3) & 0.079(4) & 0.005(3) & 0.029(3) & 0.015(4) \\ \mathrm{C}(3) & 0.062(3) & 0.094(3) & 0.077(4) & 0.020(3) & 0.036(3) & 0.016(4)\end{array}$




$\begin{array}{lllllll}\mathrm{C}(4) & 0.068(3) & 0.076(3) & 0.048(3) & 0.006(2) & 0.027(3) & 0.001(3) \\ \mathrm{C}(5) & 0.064(3) & 0.074(2) & 0.076(3) & -0.001(3) & 0.039(3) & 0.015(3) \\ \mathrm{C}(6) & 0.048(2) & 0.073(2) & 0.063(3) & 0.002(2) & 0.028(3) & 0.010(3) \\ \mathrm{C}(7) & 0.048(3) & 0.080(2) & 0.048(3) & -0.006(2) & 0.018(2) & 0.006(3) \\ \mathrm{C}(8) & 0.077(3) & 0.069(3) & 0.056(2) & -0.004(2) & 0.041(2) & -0.004(3) \\ \mathrm{C}(9) & 0.068(3) & 0.077(3) & 0.055(3) & -0.018(3) & 0.026(2) & -0.002(3) \\ \mathrm{C}(10) & 0.051(2) & 0.078(2) & 0.034(2) & -0.008(2) & 0.017(2) & 0.008(3) \\ \mathrm{C}(11) & 0.055(2) & 0.070(2) & 0.068(3) & 0.003(3) & 0.040(2) & 0.008(3) \\ \mathrm{C}(12) & 0.054(2) & 0.080(3) & 0.055(2) & -0.011(3) & 0.027(2) & -0.003(3) \\ \mathrm{C}(13) & 0.077(3) & 0.078(3) & 0.052(3) & 0.003(3) & 0.034(3) & -0.003(3) \\ \mathrm{C}(14) & 0.076(3) & 0.087(3) & 0.055(3) & 0.015(3) & 0.041(3) & 0.014(3) \\ \mathrm{C}(15) & 0.082(4) & 0.081(3) & 0.079(3) & 0.016(3) & 0.051(3) & 0.020(3) \\ \mathrm{C}(16) & 0.098(4) & 0.061(3) & 0.082(3) & 0.009(3) & 0.059(3) & 0.002(3)\end{array}$

The general temperature factor expression:

$\exp \left(-2 \mathrm{PI}^{2}\left(\mathrm{a}^{*} \mathrm{U}_{11} \mathrm{~h}^{2}+\mathrm{b}^{*} \mathrm{U}_{22} \mathrm{k}^{2}+\mathrm{c}^{* 2} \mathrm{U}_{33} \mathrm{l}^{2}+2 \mathrm{a} * \mathrm{~b}^{*} \mathrm{U}_{12} \mathrm{hk}+2 \mathrm{a}{ }^{*} \mathrm{c}^{*} \mathrm{U}_{13} \mathrm{hl}+\right.\right.$ $\left.\left.2 \mathrm{~b}^{*} \mathrm{c} * \mathrm{U}_{23} \mathrm{kl}\right)\right)$

Table S-16. Bond Lengths $(\AA)$

$\begin{array}{llllll}\text { atom } & \text { atom } & \text { distance } & \text { atom } & \text { atom } & \text { distance } \\ \mathrm{O}(1) & \mathrm{N}(1) & 1.210(5) & \mathrm{O}(2) & \mathrm{N}(1) & 1.231(5) \\ \mathrm{O}(3) & \mathrm{C}(8) & 1.414(5) & \mathrm{O}(3) & \mathrm{C}(11) & 1.432(5) \\ \mathrm{O}(4) & \mathrm{C}(8) & 1.426(5) & \mathrm{O}(4) & \mathrm{C}(12) & 1.420(5) \\ \mathrm{O}(5) & \mathrm{C}(12) & 1.401(5) & \mathrm{O}(5) & \mathrm{C}(16) & 1.427(5) \\ \mathrm{N}(1) & \mathrm{C}(4) & 1.452(6) & \mathrm{C}(1) & \mathrm{C}(2) & 1.389(6) \\ \mathrm{C}(1) & \mathrm{C}(6) & 1.398(6) & \mathrm{C}(1) & \mathrm{C}(7) & 1.454(6) \\ \mathrm{C}(2) & \mathrm{C}(3) & 1.379(6) & \mathrm{C}(2) & \mathrm{H}(1) & 1.06 \\ \mathrm{C}(3) & \mathrm{C}(4) & 1.364(6) & \mathrm{C}(3) & \mathrm{H}(2) & 1.03 \\ \mathrm{C}(4) & \mathrm{C}(5) & 1.387(6) & \mathrm{C}(5) & \mathrm{C}(6) & 1.379(6) \\ \mathrm{C}(5) & \mathrm{H}(3) & 0.99 & \mathrm{C}(6) & \mathrm{H}(4) & 1.02 \\ \mathrm{C}(7) & \mathrm{C}(10) & 1.331(6) & \mathrm{C}(7) & \mathrm{H}(5) & 1.02 \\ \mathrm{C}(8) & \mathrm{C}(9) & 1.516(6) & \mathrm{C}(8) & \mathrm{H}(6) & 1.02 \\ \mathrm{C}(9) & \mathrm{C}(10) & 1.494(6) & \mathrm{C}(9) & \mathrm{H}(7) & 0.98 \\ \mathrm{C}(9) & \mathrm{H}(8) & 0.98 & \mathrm{C}(10) & \mathrm{C}(11) & 1.506(5)\end{array}$




$\begin{array}{llllll}\mathrm{C}(11) & \mathrm{H}(9) & 1.01 & \mathrm{C}(11) & \mathrm{H}(10) & 1.02 \\ \mathrm{C}(12) & \mathrm{C}(13) & 1.513(6) & \mathrm{C}(12) & \mathrm{H}(11) & 1.04 \\ \mathrm{C}(13) & \mathrm{C}(14) & 1.516(6) & \mathrm{C}(13) & \mathrm{H}(12) & 0.99 \\ \mathrm{C}(13) & \mathrm{H}(13) & 0.98 & \mathrm{C}(14) & \mathrm{C}(15) & 1.510(6) \\ \mathrm{C}(14) & \mathrm{H}(14) & 0.96 & \mathrm{C}(14) & \mathrm{H}(15) & 0.94 \\ \mathrm{C}(15) & \mathrm{C}(16) & 1.504(7) & \mathrm{C}(15) & \mathrm{H}(16) & 0.98 \\ \mathrm{C}(15) & \mathrm{H}(17) & 0.97 & \mathrm{C}(16) & \mathrm{H}(18) & 0.95 \\ \mathrm{C}(16) & \mathrm{H}(19) & 0.98 & & & \end{array}$

Table S-17. Bond Angles $\left({ }^{\circ}\right)$

$\begin{array}{llllllll}\text { atom } & \text { atom } & \text { atom } & \text { angle } & \text { atom } & \text { atom } & \text { atom } & \text { angle } \\ & & & & & & \\ \mathrm{C}(8) & \mathrm{O}(3) & \mathrm{C}(11) & 108.4(3) & \mathrm{C}(8) & \mathrm{O}(4) & \mathrm{C}(12) & 114.3(3) \\ \mathrm{C}(12) & \mathrm{O}(5) & \mathrm{C}(16) & 113.6(3) & \mathrm{O}(1) & \mathrm{N}(1) & \mathrm{O}(2) & 121.5(6) \\ \mathrm{O}(1) & \mathrm{N}(1) & \mathrm{C}(4) & 119.4(6) & \mathrm{O}(2) & \mathrm{N}(1) & \mathrm{C}(4) & 119.1(6) \\ \mathrm{C}(2) & \mathrm{C}(1) & \mathrm{C}(6) & 117.5(5) & \mathrm{C}(2) & \mathrm{C}(1) & \mathrm{C}(7) & 118.4(4) \\ \mathrm{C}(6) & \mathrm{C}(1) & \mathrm{C}(7) & 124.1(5) & \mathrm{C}(1) & \mathrm{C}(2) & \mathrm{C}(3) & 121.7(5) \\ \mathrm{C}(1) & \mathrm{C}(2) & \mathrm{H}(1) & 117.6 & \mathrm{C}(3) & \mathrm{C}(2) & \mathrm{H}(1) & 120.6 \\ \mathrm{C}(2) & \mathrm{C}(3) & \mathrm{C}(4) & 119.8(5) & \mathrm{C}(2) & \mathrm{C}(3) & \mathrm{H}(2) & 118.9 \\ \mathrm{C}(4) & \mathrm{C}(3) & \mathrm{H}(2) & 121.2 & \mathrm{~N}(1) & \mathrm{C}(4) & \mathrm{C}(3) & 121.1(5) \\ \mathrm{N}(1) & \mathrm{C}(4) & \mathrm{C}(5) & 118.7(5) & \mathrm{C}(3) & \mathrm{C}(4) & \mathrm{C}(5) & 120.2(5) \\ \mathrm{C}(4) & \mathrm{C}(5) & \mathrm{C}(6) & 119.9(5) & \mathrm{C}(4) & \mathrm{C}(5) & \mathrm{H}(3) & 119.6 \\ \mathrm{C}(6) & \mathrm{C}(5) & \mathrm{H}(3) & 120.5 & \mathrm{C}(1) & \mathrm{C}(6) & \mathrm{C}(5) & 120.9(5) \\ \mathrm{C}(1) & \mathrm{C}(6) & \mathrm{H}(4) & 118.4 & \mathrm{C}(5) & \mathrm{C}(6) & \mathrm{H}(4) & 120.7 \\ \mathrm{C}(1) & \mathrm{C}(7) & \mathrm{C}(10) & 130.7(4) & \mathrm{C}(1) & \mathrm{C}(7) & \mathrm{H}(5) & 113.2 \\ \mathrm{C}(10) & \mathrm{C}(7) & \mathrm{H}(5) & 115.9 & \mathrm{O}(3) & \mathrm{C}(8) & \mathrm{O}(4) & 111.5(4) \\ \mathrm{O}(3) & \mathrm{C}(8) & \mathrm{C}(9) & 104.7(4) & \mathrm{O}(3) & \mathrm{C}(8) & \mathrm{H}(6) & 109.5 \\ \mathrm{O}(4) & \mathrm{C}(8) & \mathrm{C}(9) & 106.9(4) & \mathrm{O}(4) & \mathrm{C}(8) & \mathrm{H}(6) & 111.7 \\ \mathrm{C}(9) & \mathrm{C}(8) & \mathrm{H}(6) & 112.4 & \mathrm{C}(8) & \mathrm{C}(9) & \mathrm{C}(10) & 102.5(4) \\ \mathrm{C}(8) & \mathrm{C}(9) & \mathrm{H}(7) & 113.7 & \mathrm{C}(8) & \mathrm{C}(9) & \mathrm{H}(8) & 111.4 \\ \mathrm{C}(10) & \mathrm{C}(9) & \mathrm{H}(7) & 112.6 & \mathrm{C}(10) & \mathrm{C}(9) & \mathrm{H}(8) & 113.1 \\ \mathrm{H}(7) & \mathrm{C}(9) & \mathrm{H}(8) & 104.0 & \mathrm{C}(7) & \mathrm{C}(10) & \mathrm{C}(9) & 125.8(4) \\ \mathrm{C}(7) & \mathrm{C}(10) & \mathrm{C}(11) & 128.0(4) & \mathrm{C}(9) & \mathrm{C}(10) & \mathrm{C}(11) & 106.2(4) \\ \mathrm{O}(3) & \mathrm{C}(11) & \mathrm{C}(10) & 106.1(4) & \mathrm{O}(3) & \mathrm{C}(11) & \mathrm{H}(9) & 114.6 \\ \mathrm{O}(3) & \mathrm{C}(11) & \mathrm{H}(10) & 113.7 & \mathrm{C}(10) & \mathrm{C}(11) & \mathrm{H}(9) & 112.1\end{array}$




\begin{tabular}{|c|c|c|c|c|c|c|c|}
\hline $\mathrm{C}(10)$ & $\mathrm{C}(11)$ & $\mathrm{H}(10)$ & 111.1 & $\mathrm{H}(9)$ & $\mathrm{C}(11)$ & $\mathrm{H}(10)$ & 99.3 \\
\hline \multicolumn{8}{|c|}{ Table S-18. Bond Angles () (continued) } \\
\hline atom & atom & atom & angle & atom & atom & atom & angle \\
\hline $\mathrm{O}(4)$ & $\mathrm{C}(12)$ & $\mathrm{O}(5)$ & $112.3(4)$ & $\mathrm{O}(4)$ & $\mathrm{C}(12)$ & $\mathrm{C}(13)$ & $106.8(4)$ \\
\hline $\mathrm{O}(4)$ & $\mathrm{C}(12)$ & $\mathrm{H}(11)$ & 110.0 & $\mathrm{O}(5)$ & $\mathrm{C}(12)$ & $\mathrm{C}(13)$ & $113.5(4)$ \\
\hline $\mathrm{O}(5)$ & $\mathrm{C}(12)$ & $\mathrm{H}(11)$ & 108.6 & $\mathrm{C}(13)$ & $\mathrm{C}(12)$ & $\mathrm{H}(11)$ & 105.5 \\
\hline $\mathrm{C}(12)$ & $\mathrm{C}(13)$ & $\mathrm{C}(14)$ & $111.8(4)$ & $\mathrm{C}(12)$ & $\mathrm{C}(13)$ & $\mathrm{H}(12)$ & 112.7 \\
\hline $\mathrm{C}(12)$ & $\mathrm{C}(13)$ & $\mathrm{H}(13)$ & 110.8 & $\mathrm{C}(14)$ & $\mathrm{C}(13)$ & $\mathrm{H}(12)$ & 108.9 \\
\hline $\mathrm{C}(14)$ & $\mathrm{C}(13)$ & $\mathrm{H}(13)$ & 109.0 & $\mathrm{H}(12)$ & $\mathrm{C}(13)$ & $\mathrm{H}(13)$ & 103.3 \\
\hline $\mathrm{C}(13)$ & $\mathrm{C}(14)$ & $C(15)$ & $110.3(4)$ & $\mathrm{C}(13)$ & $\mathrm{C}(14)$ & $\mathrm{H}(14)$ & 110.3 \\
\hline $\mathrm{C}(13)$ & $\mathrm{C}(14)$ & $\mathrm{H}(15)$ & 108.4 & $\mathrm{C}(15)$ & $\mathrm{C}(14)$ & $\mathrm{H}(14)$ & 109.5 \\
\hline$C(15)$ & $\mathrm{C}(14)$ & $\mathrm{H}(15)$ & 108.8 & $\mathrm{H}(14)$ & $\mathrm{C}(14)$ & $\mathrm{H}(15)$ & 109.4 \\
\hline $\mathrm{C}(14)$ & $\mathrm{C}(15)$ & $\mathrm{C}(16)$ & $109.9(4)$ & $\mathrm{C}(14)$ & $\mathrm{C}(15)$ & $\mathrm{H}(16)$ & 109.8 \\
\hline $\mathrm{C}(14)$ & $\mathrm{C}(15)$ & $\mathrm{H}(17)$ & 109.6 & $\mathrm{C}(16)$ & $\mathrm{C}(15)$ & $\mathrm{H}(16)$ & 110.0 \\
\hline $\mathrm{C}(16)$ & $\mathrm{C}(15)$ & $\mathrm{H}(17)$ & 111.7 & $\mathrm{H}(16)$ & $\mathrm{C}(15)$ & $\mathrm{H}(17)$ & 105.7 \\
\hline $\mathrm{O}(5)$ & $\mathrm{C}(16)$ & $C(15)$ & $110.8(4)$ & $\mathrm{O}(5)$ & $\mathrm{C}(16)$ & $\mathrm{H}(18)$ & 112.1 \\
\hline $\mathrm{O}(5)$ & $\mathrm{C}(16)$ & $\mathrm{H}(19)$ & 110.1 & $C(15)$ & $\mathrm{C}(16)$ & $\mathrm{H}(18)$ & 108.5 \\
\hline$C(15)$ & $\mathrm{C}(16)$ & $\mathrm{H}(19)$ & 108.5 & $\mathrm{H}(18)$ & $\mathrm{C}(16)$ & $\mathrm{H}(19)$ & 106.7 \\
\hline
\end{tabular}

Table S-19. Torsion Angles $\left({ }^{\circ}\right)$

$\begin{array}{lllllllllr}\text { atom } & \text { atom } & \text { atom } & \text { atom } & \text { angle } & \text { atom } & \text { atom } & \text { atom } & \text { atom } & \text { angle } \\ \mathrm{O}(1) & \mathrm{N}(1) & \mathrm{C}(4) & \mathrm{C}(3) & 4.2(8) & \mathrm{O}(1) & \mathrm{N}(1) & \mathrm{C}(4) & \mathrm{C}(5) & -176.4(5) \\ \mathrm{O}(2) & \mathrm{N}(1) & \mathrm{C}(4) & \mathrm{C}(3) & -174.7(5) & \mathrm{O}(2) & \mathrm{N}(1) & \mathrm{C}(4) & \mathrm{C}(5) & 4.7(7) \\ \mathrm{O}(3) & \mathrm{C}(8) & \mathrm{O}(4) & \mathrm{C}(12) & -55.8(5) & \mathrm{O}(3) & \mathrm{C}(8) & \mathrm{C}(9) & \mathrm{C}(10) & -33.9(4) \\ \mathrm{O}(3) & \mathrm{C}(11) & \mathrm{C}(10) & \mathrm{C}(7) & 174.5(4) & \mathrm{O}(3) & \mathrm{C}(11) & \mathrm{C}(10) & \mathrm{C}(9) & -2.5(5) \\ \mathrm{O}(4) & \mathrm{C}(8) & \mathrm{O}(3) & \mathrm{C}(11) & -81.2(4) & \mathrm{O}(4) & \mathrm{C}(8) & \mathrm{C}(9) & \mathrm{C}(10) & 84.5(4) \\ \mathrm{O}(4) & \mathrm{C}(12) & \mathrm{O}(5) & \mathrm{C}(16) & -67.0(5) & \mathrm{O}(4) & \mathrm{C}(12) & \mathrm{C}(13) & \mathrm{C}(14) & 75.3(5) \\ \mathrm{O}(5) & \mathrm{C}(12) & \mathrm{O}(4) & \mathrm{C}(8) & -55.4(5) & \mathrm{O}(5) & \mathrm{C}(12) & \mathrm{C}(13) & \mathrm{C}(14) & -49.0(5) \\ \mathrm{O}(5) & \mathrm{C}(16) & \mathrm{C}(15) & \mathrm{C}(14) & 58.7(5) & \mathrm{N}(1) & \mathrm{C}(4) & \mathrm{C}(3) & \mathrm{C}(2) & 179.0(5) \\ \mathrm{N}(1) & \mathrm{C}(4) & \mathrm{C}(5) & \mathrm{C}(6) & -178.9(4) & \mathrm{C}(1) & \mathrm{C}(2) & \mathrm{C}(3) & \mathrm{C}(4) & 0.2(8) \\ \mathrm{C}(1) & \mathrm{C}(6) & \mathrm{C}(5) & \mathrm{C}(4) & -0.5(7) & \mathrm{C}(1) & \mathrm{C}(7) & \mathrm{C}(10) & \mathrm{C}(9) & 179.6(5)\end{array}$




$\begin{array}{lllllllllr}\mathrm{C}(1) & \mathrm{C}(7) & \mathrm{C}(10) & \mathrm{C}(11) & 3.2(8) & \mathrm{C}(2) & \mathrm{C}(1) & \mathrm{C}(6) & \mathrm{C}(5) & 0.3(7) \\ \mathrm{C}(2) & \mathrm{C}(1) & \mathrm{C}(7) & \mathrm{C}(10) & -178.2(5) & \mathrm{C}(2) & \mathrm{C}(3) & \mathrm{C}(4) & \mathrm{C}(5) & -0.4(8) \\ \mathrm{C}(3) & \mathrm{C}(2) & \mathrm{C}(1) & \mathrm{C}(6) & -0.2(8) & \mathrm{C}(3) & \mathrm{C}(2) & \mathrm{C}(1) & \mathrm{C}(7) & -179.7(5) \\ \mathrm{C}(3) & \mathrm{C}(4) & \mathrm{C}(5) & \mathrm{C}(6) & 0.6(7) & \mathrm{C}(5) & \mathrm{C}(6) & \mathrm{C}(1) & \mathrm{C}(7) & 179.8(5) \\ \mathrm{C}(6) & \mathrm{C}(1) & \mathrm{C}(7) & \mathrm{C}(10) & 2.2(8) & \mathrm{C}(7) & \mathrm{C}(10) & \mathrm{C}(9) & \mathrm{C}(8) & -155.3(4) \\ \mathrm{C}(8) & \mathrm{O}(3) & \mathrm{C}(11) & \mathrm{C}(10) & -19.8(4) & \mathrm{C}(8) & \mathrm{O}(4) & \mathrm{C}(12) & \mathrm{C}(13) & 179.6(4) \\ \mathrm{C}(8) & \mathrm{C}(9) & \mathrm{C}(10) & \mathrm{C}(11) & 21.8(5) & \mathrm{C}(9) & \mathrm{C}(8) & \mathrm{O}(3) & \mathrm{C}(11) & 34.0(4) \\ \mathrm{C}(9) & \mathrm{C}(8) & \mathrm{O}(4) & \mathrm{C}(12) & -169.6(4) & \mathrm{C}(12) & \mathrm{O}(5) & \mathrm{C}(16) & \mathrm{C}(15) & -59.2(5) \\ \mathrm{C}(12) & \mathrm{C}(13) & \mathrm{C}(14) & \mathrm{C}(15) & 49.1(5) & \mathrm{C}(13) & \mathrm{C}(12) & \mathrm{O}(5) & \mathrm{C}(16) & 54.2(5) \\ \mathrm{C}(13) & \mathrm{C}(14) & \mathrm{C}(15) & \mathrm{C}(16) & -54.1(5) & & & & & \end{array}$

Table S-20. Non-bonded Contacts out to $3.60 \AA$

$\begin{array}{llllll}\text { atom } & \text { atom } & \text { distance } & \text { atom } & \text { atom } & \text { distance } \\ \mathrm{O}(1) & \mathrm{H}(7)^{1)} & 2.92 & \mathrm{O}(1) & \mathrm{H}(17)^{2)} & 2.92 \\ \mathrm{O}(1) & \mathrm{H}(18)^{2)} & 2.99 & \mathrm{O}(1) & \mathrm{H}(6)^{3)} & 3.01 \\ \mathrm{O}(1) & \mathrm{H}(15)^{2)} & 3.02 & \mathrm{O}(1) & \mathrm{H}(18)^{3)} & 3.13 \\ \mathrm{O}(1) & \mathrm{C}(15)^{2)} & 3.484(7) & \mathrm{O}(2) & \mathrm{H}(6)^{3)} & 2.69 \\ \mathrm{O}(2) & \mathrm{H}(14)^{4)} & 2.91 & \mathrm{O}(2) & \mathrm{H}(7)^{1)} & 3.06 \\ \mathrm{O}(2) & \mathrm{H}(9)^{5)} & 3.08 & \mathrm{O}(2) & \mathrm{H}(18)^{3)} & 3.11 \\ \mathrm{O}(2) & \mathrm{H}(19)^{3)} & 3.15 & \mathrm{O}(2) & \mathrm{H}(13)^{4)} & 3.26 \\ \mathrm{O}(2) & \mathrm{H}(15)^{4)} & 3.37 & \mathrm{O}(2) & \mathrm{H}(10)^{5)} & 3.43 \\ \mathrm{O}(2) & \mathrm{C}(16)^{3)} & 3.461(7) & \mathrm{O}(2) & \mathrm{C}(14)^{4)} & 3.475(6) \\ \mathrm{O}(2) & \mathrm{C}(11)^{5)} & 3.577(6) & \mathrm{O}(3) & \mathrm{H}(3)^{6} & 2.40 \\ \mathrm{O}(3) & \mathrm{H}(17)^{7)} & 2.83 & \mathrm{O}(3) & \mathrm{H}(14)^{7)} & 2.84 \\ \mathrm{O}(3) & \mathrm{C}(5)^{6)} & 3.340(6) & \mathrm{O}(3) & \mathrm{C}(15)^{7)} & 3.413(5) \\ \mathrm{O}(3) & \mathrm{H}(16)^{7)} & 3.45 & \mathrm{O}(3) & \mathrm{C}(14)^{7} & 3.476(5) \\ \mathrm{O}(4) & \mathrm{H}(8)^{8)} & 2.74 & \mathrm{O}(5) & \mathrm{H}(1)^{9)} & 2.77 \\ \mathrm{O}(5) & \mathrm{H}(4)^{6} & 2.94 & \mathrm{O}(5) & \mathrm{H}(10)^{6)} & 3.18 \\ \mathrm{O}(5) & \mathrm{H}(3)^{6)} & 3.36 & \mathrm{O}(5) & \mathrm{C}(2)^{9)} & 3.494(5) \\ \mathrm{O}(5) & \mathrm{H}(2)^{9)} & 3.52 & \mathrm{~N}(1) & \mathrm{H}(7)^{1)} & 3.00 \\ \mathrm{~N}(1) & \mathrm{H}(18)^{3)} & 3.15 & \mathrm{~N}(1) & \mathrm{H}(6)^{3)} & 3.22 \\ \mathrm{~N}(1) & \mathrm{H}(19)^{3)} & 3.47 & \mathrm{C}(1) & \left.\mathrm{H}(1)^{8}\right) & 3.54 \\ \mathrm{C}(2) & \mathrm{H}(12)^{10)} & 3.45 & \mathrm{C}(2) & \mathrm{H}(16)^{10} & 3.56 \\ \mathrm{C}(2) & \mathrm{C}(2)^{8)} & 3.57(1) & \mathrm{H}(10)^{1)} & 3.36\end{array}$




$\begin{array}{llllll}\mathrm{C}(3) & \mathrm{H}(16)^{10)} & 3.57 & \mathrm{C}(5) & \mathrm{H}(16)^{11)} & 3.18 \\ \mathrm{C}(5) & \mathrm{H}(11)^{5)} & 3.24 & \mathrm{C}(6) & \mathrm{H}(12)^{11)} & 3.39 \\ \mathrm{C}(6) & \mathrm{H}(14)^{11)} & 3.39 & \mathrm{C}(6) & \mathrm{H}(16)^{11)} & 3.50\end{array}$

Table S-21. Non-bonded Contacts out to $3.60 \AA$ (continued)

\begin{tabular}{|c|c|c|c|c|c|}
\hline atom & atom & distance & atom & atom & distance \\
\hline $\mathrm{C}(8)$ & $H(14)^{7)}$ & 3.18 & $\mathrm{C}(8)$ & $H(3)^{6)}$ & 3.47 \\
\hline $\mathrm{C}(8)$ & $\mathrm{H}(8)^{8)}$ & 3.57 & $\mathrm{C}(9)$ & $\mathrm{H}(15)^{8)}$ & 3.15 \\
\hline $\mathrm{C}(9)$ & $\mathrm{H}(8)^{8)}$ & 3.25 & $\mathrm{C}(11)$ & $\mathrm{H}(17)^{7)}$ & 3.18 \\
\hline $\mathrm{C}(11)$ & $\mathrm{H}(3)^{6)}$ & 3.25 & $\mathrm{C}(11)$ & $\mathrm{H}(12)^{11)}$ & 3.35 \\
\hline $\mathrm{C}(12)$ & $\mathrm{H}(1)^{9)}$ & 3.05 & $\mathrm{C}(12)$ & $\mathrm{H}(3)^{6}$ & 3.30 \\
\hline $\mathrm{C}(12)$ & $\mathrm{H}(13)^{11)}$ & 3.48 & $\mathrm{C}(13)$ & $\mathrm{H}(9)^{11)}$ & 3.00 \\
\hline $\mathrm{C}(13)$ & $\mathrm{H}(1)^{9)}$ & 3.05 & $\mathrm{C}(13)$ & $\mathrm{H}(13)^{11)}$ & 3.50 \\
\hline $\mathrm{C}(14)$ & $\mathrm{H}(6)^{12)}$ & 3.39 & $\mathrm{C}(14)$ & $\mathrm{H}(7)^{8)}$ & 3.44 \\
\hline $\mathrm{C}(14)$ & $\mathrm{H}(9)^{11)}$ & 3.47 & $\mathrm{C}(14)$ & $\mathrm{H}(8)^{8)}$ & 3.54 \\
\hline $\mathrm{C}(15)$ & $\mathrm{H}(19)^{13)}$ & 3.14 & $\mathrm{C}(15)$ & $\mathrm{H}(1)^{9)}$ & 3.48 \\
\hline $\mathrm{C}(15)$ & $\mathrm{H}(2)^{14)}$ & 3.58 & $\mathrm{C}(15)$ & $\mathrm{H}(10)^{12)}$ & 3.60 \\
\hline $\mathrm{C}(16)$ & $\mathrm{H}(10)^{6)}$ & 3.45 & $\mathrm{C}(16)$ & $\mathrm{H}(1)^{9)}$ & 3.57 \\
\hline $\mathrm{C}(16)$ & $\mathrm{H}(2)^{14)}$ & 3.60 & $\mathrm{H}(1)$ & $\mathrm{H}(12)^{10)}$ & 2.40 \\
\hline $\mathrm{H}(1)$ & $\mathrm{H}(16)^{10)}$ & 2.87 & $\mathrm{H}(1)$ & $\mathrm{H}(11)^{10)}$ & 2.88 \\
\hline $\mathrm{H}(2)$ & $\mathrm{H}(17)^{2)}$ & 2.82 & $\mathrm{H}(2)$ & $H(16)^{10)}$ & 2.87 \\
\hline $\mathrm{H}(2)$ & $\mathrm{H}(10)^{1)}$ & 3.01 & $\mathrm{H}(2)$ & $H(19)^{10)}$ & 3.26 \\
\hline $\mathrm{H}(2)$ & $\mathrm{H}(19)^{2)}$ & 3.29 & $\mathrm{H}(2)$ & $\mathrm{H}(18)^{2)}$ & 3.32 \\
\hline $\mathrm{H}(3)$ & $H(11)^{5)}$ & 2.53 & $\mathrm{H}(3)$ & $\mathrm{H}(16)^{11)}$ & 3.08 \\
\hline $\mathrm{H}(3)$ & $\mathrm{H}(9)^{5)}$ & 3.28 & $\mathrm{H}(3)$ & $\mathrm{H}(14)^{11)}$ & 3.30 \\
\hline $\mathrm{H}(3)$ & $\mathrm{H}(10)^{5)}$ & 3.47 & $\mathrm{H}(3)$ & $\mathrm{H}(17)^{11)}$ & 3.58 \\
\hline $\mathrm{H}(4)$ & $\mathrm{H}(14)^{11)}$ & 2.87 & $\mathrm{H}(4)$ & $\mathrm{H}(12)^{11)}$ & 3.12 \\
\hline $\mathrm{H}(5)$ & $\mathrm{H}(11)^{10)}$ & 3.06 & $\mathrm{H}(5)$ & $\mathrm{H}(12)^{10)}$ & 3.08 \\
\hline $\mathrm{H}(5)$ & $\mathrm{H}(13)^{8)}$ & 3.23 & $\mathrm{H}(6)$ & $\mathrm{H}(14)^{7)}$ & 2.61 \\
\hline $\mathrm{H}(6)$ & $H(15)^{7)}$ & 3.40 & $\mathrm{H}(7)$ & $\mathrm{H}(15)^{8)}$ & 2.62 \\
\hline
\end{tabular}


Table S-22. Non-bonded Contacts out to $3.60 \AA$ (continued)

$\begin{array}{llllll}\text { atom } & \text { atom } & \text { distance } & \text { atom } & \text { atom } & \text { distance } \\ \mathrm{H}(7) & \mathrm{H}(13)^{8)} & 3.59 & \mathrm{H}(8) & \mathrm{H}(8)^{8)} & 2.44 \\ \mathrm{H}(8) & \mathrm{H}(15)^{8)} & 2.72 & \mathrm{H}(8) & \mathrm{H}(13)^{8)} & 3.34 \\ \mathrm{H}(9) & \mathrm{H}(12)^{11)} & 2.43 & \mathrm{H}(9) & \mathrm{H}(13)^{11)} & 2.76 \\ \mathrm{H}(9) & \mathrm{H}(14)^{11)} & 2.96 & \mathrm{H}(10) & \mathrm{H}(17)^{7)} & 2.71 \\ \mathrm{H}(10) & \mathrm{H}(19)^{5)} & 2.81 & \mathrm{H}(11) & \mathrm{H}(13)^{11)} & 2.73 \\ \mathrm{H}(11) & \mathrm{H}(12)^{11)} & 3.60 & \mathrm{H}(12) & \mathrm{H}(13)^{11)} & 3.43 \\ \mathrm{H}(13) & \mathrm{H}(13)^{11)} & 3.05 & \mathrm{H}(16) & \mathrm{H}(19)^{13)} & 2.79 \\ \mathrm{H}(17) & \mathrm{H}(19)^{13)} & 2.74 & \mathrm{H}(19) & \mathrm{H}(19)^{13)} & 3.07\end{array}$

Symmetry operations
(1) $-\mathrm{X}+1,-\mathrm{Y}+1,-\mathrm{Z}$
(2) $-\mathrm{X}+1, \mathrm{Y}+1,-\mathrm{Z}+1 / 2$
(3) $\mathrm{X}, \mathrm{Y}+1, \mathrm{Z}$
(4) $\quad \mathrm{X},-\mathrm{Y}+1, \mathrm{Z}-1 / 2$
(5) $\quad-\mathrm{X}+3 / 2, \mathrm{Y}+1 / 2,-\mathrm{Z}+1 / 2$
(6) $-\mathrm{X}+3 / 2, \mathrm{Y}-1 / 2,-\mathrm{Z}+1 / 2$
(7) $\mathrm{X},-\mathrm{Y}, \mathrm{Z}-1 / 2$
(8) $-\mathrm{X}+1, \mathrm{Y},-\mathrm{Z}+1 / 2$
(9) $\mathrm{X}+1 / 2,-\mathrm{Y}+1 / 2, \mathrm{Z}+1 / 2$
(10) $\mathrm{X}-1 / 2,-\mathrm{Y}+1 / 2, \mathrm{Z}-1 / 2$
(11) $\quad-\mathrm{X}+3 / 2,-\mathrm{Y}+1 / 2,-\mathrm{Z}+1$
(12) $\quad \mathrm{X},-\mathrm{Y}, \mathrm{Z}+1 / 2$
(13) $-\mathrm{X}+3 / 2,-\mathrm{Y}-1 / 2,-\mathrm{Z}+1$
(14) $-\mathrm{X}+1, \mathrm{Y}-1,-\mathrm{Z}+1 / 2$ 